\title{
Invertebrate Abundance, Biomass, and Richness Associated with an Exotic Invasive Shrub (Lonicera morrowii A. Gray)
}

\author{
Jason P. Love ${ }^{1,2}$, James T. Anderson ${ }^{1}$ \\ ${ }^{1}$ Division of Forestry and Natural Resources, West Virginia University, Morgantown, WV, USA \\ ${ }^{2}$ Highlands Biological Station, Western Carolina University, Highlands, NC, USA \\ Email: jim.anderson@mail.wvu.edu
}

How to cite this paper: Love, J.P. and Anderson, J.T. (2020) Invertebrate Abundance, Biomass, and Richness Associated with an Exotic Invasive Shrub (Lonicera morrowii A. Gray). Natural Resources, 11, 257-282.

https://doi.org/10.4236/nr.2020.117016

Received: May 21, 2020

Accepted: July 14, 2020

Published: July 17, 2020

Copyright $\odot 2020$ by author(s) and Scientific Research Publishing Inc. This work is licensed under the Creative Commons Attribution International License (CC BY 4.0).

http://creativecommons.org/licenses/by/4.0/

\begin{abstract}
Exotic bush honeysuckles (Lonicera spp.) are becoming increasingly common in the eastern and mid-western United States, but little is known about their impacts on invertebrates. We used a modified leaf vacuum to sample invertebrates in the shrub strata and understory of three shrub types (and open plots in the understory): single Morrow's honeysuckle (L. morrowii A. Gray) shrubs, single native southern arrowwood (Viburnum recognitum Fernald) shrubs, and dense thickets of Morrow's honeysuckle, in southwestern PA, USA during 2004 and 2005. We also assessed the degree of herbivory on the two species of shrubs. Within the shrub strata, invertebrate biomass was lower in southern arrowwood shrubs, but there was no difference in invertebrate abundance or family richness. Invertebrate abundance and richness were lowest in August, but there was no difference in biomass among the months. Invertebrate abundance, biomass, and family richness were lowest in the understory below dense thickets of Morrow's honeysuckle. Overall, the percent cover of herbs was the proximate factor responsible for driving patterns of invertebrate abundance, though ultimately these patterns were being driven by shrub type. Abundance and biomass of larval leaf chewers were highest in the native shrub; Morrow's honeysuckle had a mean of $29.7 \mathrm{~cm}^{2}$ of leaf area consumed per $1 \mathrm{~m}^{2}$ of leaf area, while the native shrub had a mean of $284.3 \mathrm{~cm}^{2}$ of leaf area consumed. Our results suggest that areas dominated by the exotic shrub negatively impact invertebrate biomass, which may in turn affect organisms at higher trophic levels.
\end{abstract}

\section{Keywords}

Invertebrate Biomass, Lonicera morrowii, Morrow's Honeysuckle, Southern Arrowwood, Terrestrial Invertebrates, Viburnum recognitum 


\section{Introduction}

Invasions of exotic plants have increased in both frequency and scale [1] so that today approximately 5000 non-native plant species have become established in the United States alone [2]. These invasions threaten biodiversity [3] [4], alter ecosystem functions [5], and impair local and global economies [2]. Several studies describe the factors that produce successful plant invasions [6] [7] [8]. The enemy release hypothesis predicts that some exotic plants are successful invaders because they lack the specialist herbivores and diseases from their native habitat, thereby conferring a competitive advantage over native plants [9] [10] [11]. Measurements of herbivore loads on native and exotic plants typically find higher numbers of invertebrates on native plants [12] [13] [14]. However, no published studies have examined patterns of invertebrate biomass on native versus exotic plants [15]. Since invasive exotic plants are becoming increasingly widespread, their impacts on invertebrates could affect members of higher trophic levels, such as insectivorous mammals, herpetofauna, and terrestrial birds. For example, $96 \%$ of terrestrial birds in North America rear their young on invertebrate protein [16]; subsequently, bird fitness is linked closely to the quality and quantity of their invertebrate food supplies [17]-[22].

Morrow's honeysuckle (Lonicera morrowii A. Gray) is an invasive exotic shrub that has spread throughout the northeastern and mid-Atlantic states [23]. The shrub is one of a suite of closely-related bush honeysuckles originally introduced into North America for horticultural purposes, including Amur honeysuckle ( $L$. maackii [Rupr.] Maxim), Tatarian honeysuckle (L. tatarica L.), and Bell's honeysuckle $(L . \times$ bella Zabel) [24] [25]. Several studies reveal that Morrow's honeysuckle and its close relatives negatively impact native herbaceous communities [4] [26] [27] [28], seedling survival [26] [27] [29], spiders [30], and vertebrates [31] [32] [33]. Additional studies describe mechanisms that may account for the shrub's success in invading foreign soils, including seed characteristics [34], seed dispersal [35] [36] [37], plant phenology [38] [39] [40] [41] [42], and allelopathy [43] [44].

Despite the relatively large body of literature on the impacts and mechanisms of bush honeysuckle invasions, no studies have addressed the impacts of the shrubs on invertebrate abundance, biomass, and diversity. The only studies that have examined invertebrate hosts of bush honeysuckles have focused their attention on the honeysuckle aphid (Hyadaphis tataricae Aizenberg, Homoptera: Aphididae), one of the few pests of bush honeysuckle [45] [46]. Examining invertebrate abundance and diversity on Morrow's honeysuckle versus native shrubs might help in our understanding of how Morrow's honeysuckle is able to successfully invade foreign regions (i.e. the enemy release hypothesis). Concurrently, invertebrate abundance and diversity may provide a measuring stick on which future restoration efforts can be assessed [47] [48] [49] [50] [51].

Little information is available about how exotic plants impact ground-dwelling invertebrates [30]. Since exotic shrub honeysuckles reduce herb richness and ab- 
undance [4] [26], the shrubs are likely to affect invertebrates found in the understory, since the type and quantity of vegetation cover strongly influences the spatial distribution of invertebrates [12] [52]. However, the shrub also may affect abiotic parameters, such as soil moisture, soil temperature, amount of leaf litter, and leaf litter nutrient content. Soil moisture [53] [54] [55] and soil temperature [56] often drive patterns of invertebrate activity, abundance, and diversity. Leaf litter quantity [53] [57] [58] and quality [57] [58] also may regulate ground-dwelling invertebrate composition and abundance.

Our objectives were to 1) compare invertebrate abundance, biomass, and diversity among single Morrow's honeysuckle shrubs, single southern arrowwood shrubs (Viburnum recognitum Fern.), and dense thickets of Morrow's honeysuckle; 2) compare ground-dwelling invertebrate abundance, biomass, and diversity among the understory of single shrubs of Morrow's honeysuckle, single shrubs of southern arrowwood, dense thickets of Morrow's honeysuckle, and open plots with no overstory; 3) determine environmental variables that drive patterns of ground-dwelling invertebrate abundance, biomass, and diversity in the understory of the four understory types; and 4) assess differences in leaf herbivory between Morrow's honeysuckle and southern arrowwood.

\section{Materials and Methods}

\subsection{Study Site}

Our study took place at Fort Necessity National Battlefield, Fayette County, southwestern Pennsylvania, USA $\left(39^{\circ} 48^{\prime} 43^{\prime \prime} \mathrm{N}, 84^{\circ} 41^{\prime} 50^{\prime \prime} \mathrm{W}\right)$ during 2004 and 2005. The park is situated in the Allegheny Mountain subregion of the Appalachian Plateau, an area also known as the southern Laurel Highlands. Our study site was a 14.6 ha meadow located in the park. The study site was formerly a mixed hardwood/conifer forest [59], but prior to 1933 the site was cleared for livestock grazing. After acquiring the land, the Park Service maintained the meadow by periodic mowing until the mid-1980s, at which time mowing ceased. It was thought that natural forest succession would eventually approximate historical vegetative conditions that existed during the 1754 battle at Fort Necessity, when George Washington and his troops unsuccessfully defended the fort against French and Indians in a battle that sparked the French-Indian War (C. Ranson, 2004, Fort Necessity National Battlefield, Farmington, PA, personal communication). However, Morrow's honeysuckle invaded the site, dominating the meadow $(67,920 \pm 4480$ shrubs/ha), and preventing regeneration of native hardwoods [42]. The site is typical of many abandoned fields in the region that have been heavily invaded by Morrow's honeysuckle and other exotic bush honeysuckle species (personal observation). Other woody shrubs and saplings found in the meadow include red maple (Acer rubrum L.), southern arrowwood, waxyfruit hawthorne (Crataegus pruinosa [Wendl. f.] K. Koch), black cherry (Prunus serotina Ehrh.), and sweet crabapple (Malus coronaria [L.] P. Mill.). Common herbaceous species include sweet vernal grass (Anthoxanthum odoratum L.), wrin- 
kleleaf goldenrod (Solidago rugosa P. Mill.), early goldenrod (S. juncea Ait.), northern dewberry (Rubus flagellaris Willd.), and orchard grass (Dactylis glomerata L.) [42].

Low lying areas are characterized by Philo silt loams. These soils are deep, poor to moderately drained, medium textured, and were formed from acidic sediments derived from sandstone and shale. Upland sites within the meadow consist of Brinkerton and Armagh silt loams, Cavode silt loams, and Gilpin channery silt loams. These soils are moderately deep, moderate to well drained, medium-textured, and underlain by acidic shale and sandstone bedrock [60].

The climate is moderate continental. The average annual temperature is $9^{\circ} \mathrm{C}$, with a mean winter temperature of $-3^{\circ} \mathrm{C}$ and a mean summer temperature of $22^{\circ} \mathrm{C}$. Average annual precipitation is $119 \mathrm{~cm}$ (Fort Necessity National Battlefield, 1991, General Management Plan/Development Concept Plan/Interpretive Prospectus, Unpublished report of Fort Necessity National Battlefield, Farmington, PA).

\subsection{Invertebrate Sampling and Identification}

Prior to sampling, we selected 45 Morrow's honeysuckle and 45 southern arrowwood shrubs (both species are in the family Caprifoliaceae) that were $\geq 1.3 \mathrm{~m}$ in height and $>2 \mathrm{~m}$ from another woody shrub. Additionally, we selected 45 sites where dense thickets of Morrow's honeysuckle were growing. One-third of the shrubs from each of the three shrub types were randomly selected without replacement to determine shrubs to be sampled for each of three sampling periods: 7-11 July 2004, 26-31 May 2005, and 1-4 August 2005. Fifteen open plots containing no shrub cover were randomly selected and evenly paired with the three shrub types and sampling period (i.e. five open plots per shrub type and sampling period). To determine the location of the open plot, a cardinal direction was randomly selected; we followed this direction from the paired shrub until an open area was found that was $>3 \mathrm{~m}$ from another shrub.

We used a modified vacuum-blower (STIHL model SH 85 D Shredder Vacuum/Blower) to sample invertebrates [61]. This method was found to be superior to sweep netting in scrub/shrub habitat [62]. We collected invertebrates from two different strata: 1) on and within the shrubs, and 2) in the understory below the shrubs. We vacuumed shrubs for one minute in a steady up and down motion while slowly circling the shrub [63]. The $1.3 \mathrm{~m}$ minimum shrub height requirement ensured that shrubs were large enough so that portions of the shrubs would not be resampled before time expired. We vacuumed $>0.5 \mathrm{~m}$ above the base of the shrub to avoid sampling invertebrates found on understory plants. When sampling invertebrates in the understory, we used a $23 \mathrm{~cm}$ diameter steel cylinder with sides $30.5 \mathrm{~cm}$ in height to delineate plots. For plots with a shrub overstory, the cylinder was placed midway between the base of the shrub and the outer perimeter of the shrub canopy. The area inside the cylinder was vacuumed for $30 \mathrm{sec}$. We sampled on relatively calm days with no rain and sampled after the dew had evaporated from the shrubs and herbs to make it easier to extract 
samples from the collection bag.

We placed the contents of each sample in a plastic bag and kept the samples on ice until the specimens could be placed in a freezer for storage. We hand-sorted invertebrates from debris under a dissecting microscope; invertebrates $\geq 2 \mathrm{~mm}$ were identified to the lowest taxonomic group (typically Family level) [64] [65] and measured to the nearest $0.1 \mathrm{~mm}$ using an ocular micrometer. We classified holometabolous insects as adults, larvae, or pupae. Ametabolous or hemimetabolous nymphs were classified as adults, since nymphs we encountered changed little as they matured, except in size and proportions. We used previously developed length-weight regression formulas to estimate dry mass of each specimen [66]-[72]. Because length-weight regression equations were not available for insect pupae, we excluded insect pupae from our biomass estimations (shrub pupae, $\mathrm{n}=4$; understory pupae, $\mathrm{n}=11$ ). After identification, we stored specimens in $70 \%$ ethanol and placed voucher specimens in the West Virginia University Division of Forestry and Natural Resources.

\subsection{Factors Influencing Ground-Dwelling Invertebrates}

We measured several biotic and abiotic variables at each understory plot after reviewing the literature for factors that influence ground-dwelling invertebrate loads. We identified and measured percent cover of herbaceous cover contained within each understory plot; nomenclature follows [73]. Additionally, we noted whether plants were native or exotic. Because the number of invertebrates might be related to the amount of debris (leaf litter and live plant material) vacuumed into the collection bag while sampling, we weighed debris vacuumed from each plot (wet weight). After vacuuming each plot for invertebrates, the remaining leaf litter was hand-collected in the area delineated by the cylinder. This litter was later added to the leaf litter (minus the live plant material) collected while vacuuming. We dried the litter at $60^{\circ} \mathrm{C}$ for $72 \mathrm{hrs}$. and then weighed it to obtain dry mass. We then ground the litter in a Wiley mill fitted with a $1 \mathrm{~mm}$ sieve; percent nitrogen was determined using the automated Kjeldahl method [74]. Afterwards we used a spade to extract the soil within the plots to a depth of about $20 \mathrm{~cm}$; soil was thoroughly hand-mixed and a sub-sample was placed in a plastic bag. We sieved the soil and a $20 \mathrm{~g}$ sub-sample was weighed, dried at $60^{\circ} \mathrm{C}$ for $>48$ hrs., then weighed again to derive percent moisture content. We used a soil temperature probe to determine soil temperature at a depth of $4 \mathrm{~cm}$.

\subsection{Leaf Herbivory}

We collected one live $35-40 \mathrm{~cm}$ long branch located $1.3 \mathrm{~m}$ above the base of each of the single Morrow's honeysuckle and southern arrowwood shrubs sampled for invertebrates. Branches were placed in a plant press and the leaves were later analyzed for herbivory. We measured herbivory using three metrics. For the first metric, we placed leaves in two categories: 1) evidence of herbivory, and 2) no evidence of herbivory. Comparisons of herbivory between shrubs were based on the number of leaves with evidence of herbivory divided by the total 
number of leaves. For the second metric, we estimated the amount of leaf area consumed $\left(\mathrm{cm}^{2}\right)$ by invertebrate herbivores using software that determines leaf area based on the number of pixels within a polygon (ImageJ version $1.33 \mathrm{u}$ : [75]). From each branch, we randomly selected $\leq 15$ leaves that showed evidence of herbivory and $\leq 15$ leaves with no evidence of herbivory. We used a digital camera (Kodak Easyshare LS443, 4.0 megapixels) to photograph the leaves. We determined the total area and amount of herbivory of each leaf to the nearest 0.1 $\mathrm{mm}^{2}$ with ImageJ software. Leaf area loss was determined by dividing the total leaf area by the area of leaf loss. For the third metric, we examined all leaves and ranked the herbivory from 0 - 5 based on visual estimation: $0=$ no herbivory; 1 $=1 \%-5 \%$ herbivory; $2=6 \%-25 \%$ herbivory; $3=26 \%-50 \%$ herbivory; $4=51 \%$ $-75 \%$ herbivory, and $5=76 \%-100 \%$ herbivory.

\subsection{Statistical Analyses}

When analyzing invertebrates, shrub species, and sample period (months) were the independent variables, while invertebrate abundance, biomass, and richness were the dependent variables. We used analysis of variance (ANOVA) (PROC GLM, SAS version 9.1; SAS Institute, Inc., Cary, NC, USA) to assess differences among shrub types, sample month, and to determine whether shrub type effects differed among sample months; Duncan's multiple range tests were used to compare differences between pairs. Invertebrate data were tested for normality and homogeneity of variance; assumptions were met using the following transformations: shrub invertebrates-eighth-root (abundance), quarter-root (biomass), and square-root (richness); understory invertebrates-square-root (abundance), quarter-root (biomass), and square-root $(x+1)$ (richness).

We used information-theory to determine biotic and abiotic factors driving patterns of ground-dwelling invertebrate abundance and biomass [76]. We tested all dependent (invertebrate abundance and biomass) and independent variables (biotic and abiotic parameters) for normality and homogeneity of variance. We used the following transformations to meet assumptions prior to data analysis: square-root-invertebrate abundance (all months), invertebrate biomass (May), soil moisture (May), litter nitrogen (August), wet debris weight (August); quarter-root-invertebrate biomass (July and August), dry litter mass (all months), wet debris weight (May and July); and arcsine-percent native herbaceous cover (July and August). We ranked (PROC RANK, SAS version 9.1) soil temperature (May and July) and soil moisture (July) data; these data did not meet assumptions even after transformations. Data transformed into ranks are thought more likely to satisfy assumptions of the parametric model than would the original non-normal data [77]. We reviewed relevant literature to specify sets of a priori candidate models for explaining ground-dwelling invertebrate abundance and biomass. We specified seven models; a global model containing all eight parameters and a subset of models representing potential influences of biotic and abiotic factors on ground-dwelling invertebrates (Tables 1-3). Following model specification, we searched for redundant variables (Spearman's $r \geq 0.70$ ) 
Table 1. Biotic and abiotic habitat parameters, measured in the understory of a degraded meadow, included in linear regression models explaining microhabitat relationships of invertebrate abundance and biomass at Fort Necessity National Battlefield, Pennsylvania, USA.

\begin{tabular}{|c|c|c|c|}
\hline Variable & Units & Abbreviation & Additional description \\
\hline Shrub type & Categorical & SH & $\begin{array}{l}\text { Morrow's honeysuckle, southern } \\
\text { arrowwood, dense thickets of Morrow's } \\
\text { honeysuckle, or open plots }\end{array}$ \\
\hline Herb cover & $\%$ & $\mathrm{HC}$ & $\%$ Total herb cover within the plot \\
\hline Native herb cover & $\%$ & NC & Ratio of native herb cover to total herb cover \\
\hline Litter mass & $\mathrm{g}$ & LM & Leaf litter dry weight within plot \\
\hline Litter nitrogen & $\%$ & $\mathrm{LN}$ & $\%$ Litter nitrogen within plot \\
\hline Soil moisture & $\%$ & SM & $\begin{array}{l}\% \text { Moisture content of } 20 \mathrm{~g} \text { sample of } \\
\text { soil from plot }\end{array}$ \\
\hline Soil temperature & ${ }^{\circ} \mathrm{C}$ & ST & Soil temperature to a depth of $4 \mathrm{~cm}$ \\
\hline Debris mass & g & $\mathrm{DM}$ & $\begin{array}{l}\text { Wet weight of debris collected } \\
\text { in vacuum within plot }\end{array}$ \\
\hline
\end{tabular}

Table 2. Linear regression models explaining influence of biotic and abiotic environmental variables on patterns of invertebrate abundance under different shrub types during different months in a degraded meadow at Fort Necessity National Battlefield, Pennsylvania, USA. Model rankings were based on Akaike's Information Criterion corrected for small sample size $\left(\mathrm{AIC}_{\mathrm{c}}\right)$.

\begin{tabular}{|c|c|c|c|c|c|}
\hline Model $^{\mathrm{a}}$ & $\mathrm{R}^{2}$ & $\mathrm{~K}^{\mathrm{b}}$ & $(\mathrm{Q}) \mathrm{AIC}_{\mathrm{c}}^{\mathrm{c}}$ & $\Delta(\mathrm{Q}) \mathrm{AIC}_{\mathrm{c}}^{\mathrm{d}}$ & $\mathrm{w}_{\mathrm{i}}^{\mathrm{e}}$ \\
\hline \multicolumn{6}{|l|}{ May $(n=59)$} \\
\hline Shrub (SH) & 0.36 & 7 & 154.82 & 0.00 & 0.99 \\
\hline Debris (DM) & 0.09 & 4 & 164.96 & 10.14 & 0.01 \\
\hline Native herbs (NC) & 0.03 & 4 & 168.91 & 14.10 & 0.00 \\
\hline Total herbs (HC) & 0.03 & 4 & 169.07 & 14.25 & 0.00 \\
\hline Litter (LM, LN) & 0.05 & 5 & 170.17 & 15.35 & 0.00 \\
\hline Soil (SM, ST) & 0.01 & 5 & 172.06 & 17.24 & 0.00 \\
\hline Global (SH, HC, NC, LM, LN, SM, ST, DM) & 0.38 & 14 & 172.56 & 17.74 & 0.00 \\
\hline \multicolumn{6}{|l|}{ July $(n=50)$} \\
\hline Total herbs (HC) & 0.26 & 3 & 136.55 & 0.00 & 0.97 \\
\hline Debris (DM) & 0.13 & 3 & 144.80 & 8.25 & 0.02 \\
\hline Shrub (SH) & 0.25 & 6 & 144.85 & 8.30 & 0.02 \\
\hline Soil (SM, ST) & 0.08 & 4 & 149.84 & 13.29 & 0.00 \\
\hline Native herbs (NC) & 0.03 & 3 & 150.12 & 13.57 & 0.00 \\
\hline Litter (LM, LN) & 0.06 & 4 & 150.92 & 14.38 & 0.00 \\
\hline Global (SH, HC, NC, LM, LN, SM, ST, DM) & 0.43 & 13 & 153.20 & 16.65 & 0.00 \\
\hline \multicolumn{6}{|l|}{ August $(\mathrm{n}=58)$} \\
\hline Total herbs (HC) & 0.03 & 3 & 181.67 & 0.00 & 0.40 \\
\hline Debris (DM) & 0.01 & 3 & 182.72 & 1.04 & 0.24 \\
\hline Native herbs (NC) & 0.01 & 3 & 183.00 & 1.33 & 0.21 \\
\hline Soil (SM, ST) & 0.01 & 4 & 185.00 & 3.33 & 0.08 \\
\hline
\end{tabular}




\section{Continued}

\begin{tabular}{cccccc}
\hline Litter (LM, LN) & $<0.01$ & 4 & 185.51 & 3.84 & 0.06 \\
Shrub (SH) & 0.03 & 6 & 188.89 & 7.21 & 0.01 \\
Global (SH, HC, NC, LM, LN, SM, ST, DM) & 0.12 & 13 & 203.72 & 22.04 & 0.00 \\
\hline
\end{tabular}

${ }^{a} \mathrm{Abbreviations} \mathrm{in} \mathrm{parentheses} \mathrm{correspond} \mathrm{to} \mathrm{model} \mathrm{parameters} \mathrm{in} \mathrm{Table} \mathrm{1.}{ }^{\mathrm{b}} \mathrm{Number}$ of estimable parameters in approximating model. For May, there is one extra parameter added to take into account the estimation of $c$, the variance inflation factor. ${ }^{~}$ In May, we used $\mathrm{QAIC}_{c}$; for July and August we used $\mathrm{AIC}_{c}{ }^{\mathrm{d}}$ Difference in value between $\mathrm{AIC}_{\mathrm{c}}$ (or $\mathrm{QAIC}_{\mathrm{c}}$ ) of the current model versus the best-approximating model $\left(\mathrm{AIC}_{\mathrm{cmin}}\right){ }^{\mathrm{e}} \mathrm{A}$ kaike weight. Probability that the current model (i) is the best-approximating model among those considered.

Table 3. Linear regression models explaining influence of biotic and abiotic environmental variables on patterns of invertebrate biomass under different shrub types during different months in a degraded meadow at Fort Necessity National Battlefield, Pennsylvania, USA. Model rankings were based on Akaike's Information Criterion corrected for small sample size $\left(\mathrm{AIC}_{\mathrm{c}}\right)$.

\begin{tabular}{|c|c|c|c|c|c|}
\hline Model $^{\mathrm{a}}$ & $\mathrm{R}^{2}$ & $\mathrm{~K}^{\mathrm{b}}$ & $\mathrm{AIC}_{\mathrm{c}}$ & $\Delta \mathrm{AIC}_{\mathrm{c}}^{\mathrm{c}}$ & $\mathrm{w}_{\mathrm{i}}^{\mathrm{d}}$ \\
\hline \multicolumn{6}{|l|}{ May $(\mathrm{n}=59)$} \\
\hline Total herbs (HC) & 0.04 & 3 & 194.44 & 0.00 & 0.47 \\
\hline Debris (DM) & 0.01 & 3 & 196.29 & 1.85 & 0.19 \\
\hline Native herbs (NC) & $<0.01$ & 3 & 196.95 & 2.51 & 0.13 \\
\hline Litter (LM, LN) & 0.03 & 4 & 197.53 & 3.09 & 0.10 \\
\hline Shrub (SH) & 0.09 & 6 & 198.81 & 4.37 & 0.05 \\
\hline Soil (SM, ST) & 0.01 & 4 & 198.91 & 4.47 & 0.05 \\
\hline Global (SH, HC, NC, LM, LN, SM, ST, DM) & 0.16 & 13 & 214.69 & 20.25 & 0.00 \\
\hline \multicolumn{6}{|l|}{ July $(\mathrm{n}=50)$} \\
\hline Total herbs (HC) & 0.16 & 3 & 55.47 & 0.00 & 0.81 \\
\hline Debris (DM) & 0.10 & 3 & 59.03 & 3.56 & 0.14 \\
\hline Native herbs (NC) & 0.03 & 3 & 62.57 & 7.10 & 0.02 \\
\hline Shrub (SH) & 0.13 & 6 & 64.42 & 8.95 & 0.01 \\
\hline Litter (LM, LN) & 0.04 & 4 & 64.59 & 9.12 & 0.01 \\
\hline Soil (SM, ST) & 0.04 & 4 & 64.64 & 9.17 & 0.01 \\
\hline Global (SH, HC, NC, LM, LN, SM, ST, DM) & 0.26 & 13 & 78.90 & 13.84 & 0.00 \\
\hline \multicolumn{6}{|l|}{ August $(\mathrm{n}=58)$} \\
\hline Shrub (SH) & 0.15 & 6 & 105.04 & 0.00 & 0.47 \\
\hline Native herbs (NC) & 0.01 & 3 & 107.05 & 2.00 & 0.17 \\
\hline Debris (DM) & $<0.01$ & 3 & 107.56 & 2.52 & 0.13 \\
\hline Total herbs (HC) & $<0.01$ & 3 & 107.59 & 2.54 & 0.13 \\
\hline Litter (LM, LN) & 0.01 & 4 & 109.41 & 4.37 & 0.05 \\
\hline Soil (SM, ST) & $<0.01$ & 4 & 109.87 & 4.83 & 0.04 \\
\hline Global (SH, HC, NC, LM, LN, SM, ST, DM) & 0.17 & 13 & 124.09 & 19.05 & 0.00 \\
\hline
\end{tabular}

${ }^{\mathrm{a}} \mathrm{Abbreviations} \mathrm{in} \mathrm{parentheses} \mathrm{correspond} \mathrm{to} \mathrm{model} \mathrm{parameters} \mathrm{in} \mathrm{Table} 1 .{ }^{\mathrm{b}}$ Number of estimable parameters in approximating model. ' $\mathrm{Difference}$ in value between $\mathrm{AIC}_{\mathrm{c}}$ of the current model versus the best-approximating model $\left(\mathrm{AIC}_{\mathrm{cmin}}\right){ }^{\mathrm{d}}$ Akaike weight. Probability that the current model (i) is the best-approximating model among those considered. 
to assess whether our models could be simplified; we found no significant correlations among the variables that we measured, so we retained eight variables for inclusion in the models. We used linear regression (PROC GENMOD, SAS version 9.1) to analyze the model set for invertebrate abundance and biomass for each of the three months separately. We checked for overdispersion within our data sets by assessing the goodness-of-fit chi-square statistic of the global models divided by their degrees of freedom (i.e. estimated single variance inflation factor, $\hat{c})$, following the protocols of [76]. Invertebrate abundance in May was overdispersed ( $\hat{c}=1.16, \mathrm{P}<0.15$ ), so we corrected for the overdispersed data using quasi-likelihood modifications (i.e. QAICc) [76]. No distinct lack of fit was found in the other data sets.

Because the number of plots sampled $(\mathrm{n} \leq 59)$ was small relative to the number of parameters $(\mathrm{K})$ (i.e. $\mathrm{n} / \mathrm{K}<40$ ), we used Akaike's Information Criterion corrected for small sample size (AICc and QAICc) for model selection [76]. We used formulas presented in [76] to calculate AICc from our maximum likelihood methods. We ranked all models according to their AICc values; the best model (i.e. the most parsimonious) was the model with the smallest AICc value [76]. We drew primary inference from models within two units of AICcmin, although models within four to seven units may have some empirical support [76]. We calculated Akaike weights (wi) to determine the strength of evidence in favor of each model and to estimate the relative importance of individual parameters [76].

When analyzing leaf herbivory data, shrub species and sample period (months) were the independent variables; percent of leaves with evidence of herbivory, leaf area consumed, and herbivory ranks were the dependent variables. We used analysis of variance (ANOVA) (PROC GLM, SAS version 9.1) to assess differences of leaf herbivory between Morrow's honeysuckle and southern arrowwood, among months, and to determine shrub $\times$ month interaction effects; we used Duncan's multiple range tests to compare differences in herbivory between shrubs and among months. We tested all leaf herbivory data for normality and homogeneity of variance; data were not normal even after transformations, so we ranked the data (PROC RANK, SAS version 9.1) prior to analysis. Untransformed means and SEs are reported throughout the results.

\section{Results}

\subsection{Invertebrates}

We collected 3133 invertebrates from the shrub strata of lone Morrow's honeysuckle shrubs, lone southern arrowwood shrubs, and dense thickets of Morrow's honeysuckle. We identified 3 Classes, 16 Orders, and 129 Families of invertebrates [42]. Composition of invertebrates based on the most abundant families and the highest biomasses differed among the three shrub types (Table 4). Total invertebrate biomass was lower in lone southern arrowwood shrubs $(\mathrm{F}=3.24$; $\mathrm{df}$ $=2,126 ; \mathrm{P}=0.043$ ), but there was no difference in invertebrate abundance or 
Table 4. The 5 most common invertebrates ( $\geq 2 \mathrm{~mm}$ in length) collected from the shrub strata of 3 shrub types ( $\mathrm{n}=45)$ based on abundance (total number of invertebrates per Family) and biomass (total dry weight (mg)) at Fort Necessity National Battlefield, Pennsylvania, USA.

\begin{tabular}{|c|c|c|c|c|c|c|c|c|c|c|}
\hline \multirow[b]{2}{*}{ Shrub type } & \multicolumn{5}{|c|}{ Top 5 based on total abundance } & \multicolumn{5}{|c|}{ Top 5 based on total biomass (mg) } \\
\hline & Order & Family & $\mathrm{n}$ & wt. & $\mathrm{F}^{\mathrm{a}}$ & Order & Family $^{\mathrm{b}}$ & wt. & $\mathrm{n}$ & $\mathrm{F}$ \\
\hline \multirow{4}{*}{$\begin{array}{c}\text { Lone Morrow’s } \\
\text { honeysuckle shrubs }\end{array}$} & Coleoptera & Staphylinidae & 72 & 53.2 & 6 & Hymentoptera & Apidae & 136.1 & 3 & 2 \\
\hline & Hymenoptera & Formicidae & 58 & 20.3 & 19 & Homoptera & Cicadellidae & 130.4 & 267 & 40 \\
\hline & Homoptera & Psyllidae & 58 & 22.3 & 16 & Hemiptera & Pentatomidae & 122.2 & 27 & 16 \\
\hline & Diptera & Chironomidae & 40 & 10.0 & 8 & Orthoptera & Gryllidae & 110.6 & 14 & 10 \\
\hline \multirow{4}{*}{$\begin{array}{l}\text { Lone southern } \\
\text { arrowwood shrubs }\end{array}$} & Hymenoptera & Formicidae & 292 & 121.8 & 32 & Coleoptera & Coccinellidae & 266.0 & 17 & 13 \\
\hline & Homoptera & Cicadellidae & 106 & 87.3 & 32 & Hemiptera & Pentatomidae & 124.9 & 33 & 10 \\
\hline & Diptera & Chironomidae & 67 & 17.8 & 7 & Hymenoptera & Symphyta & 98.9 & 28 & 14 \\
\hline & Homoptera & Aphididae & 62 & 13.0 & 5 & Homoptera & Cicadellidae & 87.3 & 106 & 32 \\
\hline \multirow{5}{*}{$\begin{array}{c}\text { Dense thickets of } \\
\text { Morrow's honeysuckle }\end{array}$} & Homoptera & Cicadellidae & 139 & 78.0 & 40 & Opiliones & Phalangiidae & 171.3 & 27 & 22 \\
\hline & Diptera & Drosophilidae & 59 & 16.4 & 21 & Coleoptera & Chrysomelidae & 131.2 & 19 & 10 \\
\hline & Hemiptera & Miridae & 47 & 33.1 & 22 & Hemiptera & Pentatomidae & 130.4 & 23 & 14 \\
\hline & Hemiptera & Reduviidae & 41 & 75.6 & 20 & Orthoptera & Acrididae & 129.0 & 14 & 8 \\
\hline & Coleoptera & Staphylinidae & 34 & 29.3 & 9 & Hemiptera & Coreidae & 96.9 & 7 & 6 \\
\hline
\end{tabular}

${ }^{\mathrm{a}}$ Frequency (number of occurrences out of 45). ${ }^{\mathrm{b}}$ Symphyta is a suborder; these specimens were also larvae.

richness among the three shrub types $(\mathrm{F} \leq 0.94 ; \mathrm{df}=2,126$; $\mathrm{P} \geq 0.394)$. Invertebrate abundance and richness was lowest in August ( $\mathrm{F} \geq 11.37$; $\mathrm{df}=2,126 ; \mathrm{P}<$ $0.001)$, but there was no difference in biomass among months $(\mathrm{F}=2.85 ; \mathrm{df}=2$, 126; $\mathrm{P}=0.062$ ). There were no shrub type $\times$ month interaction effects for invertebrate abundance, biomass, or richness $(\mathrm{F} \leq 0.92 ; \mathrm{df}=4,126 ; \mathrm{P} \geq 0.456)$ (Table 5). Larval leaf chewers (i.e. Lepidoptera and Symphyta larvae) were lowest in dense thickets of Morrow's honeysuckle ( $\mathrm{n}=11$, biomass $=30.9 \mathrm{mg}$ ), followed by lone Morrow's honeysuckle shrubs $(\mathrm{n}=16$, biomass $=107.9 \mathrm{mg})$ and lone southern arrowwood shrubs ( $\mathrm{n}=54$, biomass $=153.8 \mathrm{mg}$ ).

We collected 2589 invertebrates from the understory below lone Morrow's honeysuckle shrubs, lone southern arrowwood shrubs, dense thickets of Morrow's honeysuckle, and in open plots with no overstory. We identified 6 Classes, 17 Orders, and 115 Families of invertebrates [42]. Composition of invertebrates below the four shrub types differed based on abundance and biomass (Table 6). Invertebrate abundance, biomass, and richness were lowest in the understory below dense thickets of Morrow's honeysuckle $(\mathrm{F} \geq 5.75$; $\mathrm{df}=3,168$; $\mathrm{P}<0.001)$. Invertebrate abundance and richness were lowest in August $(\mathrm{F} \geq 13.84 ; \mathrm{df}=2$, 168; $\mathrm{P}<0.001)$, but there was no difference in invertebrate biomass among the three months $(\mathrm{F}=0.75 ; \mathrm{df}=2,168 ; \mathrm{P}=0.476)$. We found a significant shrub type 
Table 5. Mean $( \pm \mathrm{SE})$ abundance, biomass (dry weight $(\mathrm{mg})$ ), and richness (Family or lowest taxonomic group) of invertebrates ( $\geq 2 \mathrm{~mm}$ in length) sampled within the shrub strata based on shrub type and month at Fort Necessity National Battlefield, Pennsylvania, USA.

\begin{tabular}{|c|c|c|c|c|}
\hline \multirow{2}{*}{ Variable } & \multirow{2}{*}{$\mathrm{n}$} & Abundance & Biomass (mg) & Richness \\
\hline & & $\bar{X} \pm \mathrm{SE}^{*}$ & $\bar{X} \pm \mathrm{SE}$ & $\bar{X} \pm \mathrm{SE}$ \\
\hline \multicolumn{5}{|l|}{ Shrub type } \\
\hline Lone Morrow's honeysuckle shrubs & 45 & $24.0 \pm 2.2 \mathrm{~A}$ & $41.8 \pm 6.3 \mathrm{~A}$ & $10.7 \pm 0.7 \mathrm{~A}$ \\
\hline Lone southern arrowwood shrubs & 45 & $25.8 \pm 3.0 \mathrm{~A}$ & $28.3 \pm 3.8 \mathrm{~B}$ & $10.3 \pm 0.7 \mathrm{~A}$ \\
\hline Dense thickets of Morrow's honeysuckle & 45 & $19.9 \pm 1.6 \mathrm{~A}$ & $38.8 \pm 4.2 \mathrm{~A}$ & $11.0 \pm 0.6 \mathrm{~A}$ \\
\hline \multicolumn{5}{|l|}{ Month } \\
\hline May & 45 & $26.4 \pm 3.0 \mathrm{~A}$ & $30.6 \pm 4.1 \mathrm{~A}$ & $10.5 \pm 0.6 \mathrm{~B}$ \\
\hline July & 45 & $26.8 \pm 1.8 \mathrm{~A}$ & $39.2 \pm 3.3 \mathrm{~A}$ & $13.2 \pm 0.6 \mathrm{~A}$ \\
\hline August & 45 & $16.4 \pm 1.5 \mathrm{~B}$ & $39.2 \pm 6.7 \mathrm{~A}$ & $8.3 \pm 0.6 \mathrm{C}$ \\
\hline
\end{tabular}

*Means in columns with different letters and under different variables are significantly different $(\mathrm{P}<0.05)$, based on Duncan's multiple range tests.

Table 6. The 5 most common invertebrates ( $\geq 2 \mathrm{~mm}$ in length) collected from the understory of 4 shrub types $(\mathrm{n}=45)$ based on abundance (total number of invertebrates per Family) and biomass (total dry weight (mg)) at Fort Necessity National Battlefield, Pennsylvania, USA.

\begin{tabular}{|c|c|c|c|c|c|c|c|c|c|c|}
\hline \multirow{2}{*}{ Shrub type } & \multicolumn{5}{|c|}{ Top 5 based on total abundance } & \multicolumn{5}{|c|}{ Top 5 based on total biomass (mg) } \\
\hline & Order & Family $^{\mathrm{a}}$ & $\mathrm{n}$ & wt. & $\mathrm{F}^{\mathrm{b}}$ & Order & Family $^{\mathrm{a}}$ & wt. & $\mathrm{n}$ & $\mathrm{F}$ \\
\hline \multirow{5}{*}{$\begin{array}{l}\text { Lone Morrow’s } \\
\text { honeysuckle shrubs }\end{array}$} & Collembola & Isotomidae & 185 & 14.0 & 37 & Stylommatophora & - & 80.6 & 13 & 8 \\
\hline & Homoptera & Cicadellidae & 130 & 49.7 & 35 & Opiliones & Phalangiidae & 73.4 & 11 & 12 \\
\hline & Hymenoptera & Formicidae & 59 & 14.5 & 26 & Hemiptera & Pentatomidae & 59.3 & 4 & 3 \\
\hline & Coleoptera & Chrysomelidae & 30 & 2.4 & 14 & Coleoptera & Chrysomelidae & 54.2 & 18 & 17 \\
\hline & Coleoptera & Curculionidae & 27 & 29.3 & 13 & Homoptera & Cicadellidae & 49.7 & 130 & 35 \\
\hline \multirow{5}{*}{$\begin{array}{l}\text { Lone southern } \\
\text { arrowwood shrubs }\end{array}$} & Homoptera & Cicadellidae & 160 & 83.4 & 36 & Homoptera & Cicadellidae & 83.4 & 160 & 36 \\
\hline & Collembola & Isotomidae & 145 & 12.7 & 29 & Opiliones & Phalangiidae & 71.9 & 7 & 6 \\
\hline & Hymenoptera & Formicidae & 67 & 17.7 & 27 & Stylommatophora & - & 50.6 & 9 & 8 \\
\hline & Araneae & Lycosidae & 28 & 39.9 & 14 & Coleoptera & Chrysomelidae & 48.1 & 10 & 9 \\
\hline & Coleoptera & Curculionidae & 28 & 23.3 & 18 & Araneae & Lycosidae & 39.9 & 28 & 14 \\
\hline \multirow{5}{*}{$\begin{array}{l}\text { Dense thickets of } \\
\text { Morrow's honeysuckle }\end{array}$} & Collembola & Isotomidae & 106 & 8.1 & 28 & Hemiptera & Pentatomidae & 85.0 & 9 & 7 \\
\hline & Homoptera & Cicadellidae & 44 & 20.0 & 18 & Stylommatophora & - & 72.1 & 13 & 12 \\
\hline & Hymenoptera & Formicidae & 38 & 11.6 & 17 & Coleoptera & Curculionidae & 30.8 & 23 & 14 \\
\hline & Coleoptera & Curculionidae & 23 & 30.8 & 14 & Homoptera & Cicadellidae & 20.0 & 44 & 18 \\
\hline & Diptera & Sciaridae & 17 & 3.8 & 6 & Araneae & Thomasidae & 20.0 & 1 & 1 \\
\hline \multirow{5}{*}{$\begin{array}{l}\text { Open plots with } \\
\text { no overstory }\end{array}$} & Homoptera & Cicadellidae & 195 & 97.7 & 37 & Homoptera & Cicadellidae & 97.7 & 195 & 37 \\
\hline & Collembola & Isotomidae & 152 & 8.4 & 31 & Araneae & Lycosidae & 65.6 & 31 & 16 \\
\hline & Hymenoptera & Formicidae & 70 & 37.2 & 21 & Stylommatophora & - & 45.4 & 7 & 7 \\
\hline & Coleoptera & Chrysomelidae & 54 & 4.0 & 10 & Lepidoptera & Noctuidae & 38.8 & 10 & 5 \\
\hline & Homoptera & Psyllidae & 44 & 15.2 & 16 & Hymenoptera & Formicidae & 37.2 & 70 & 21 \\
\hline
\end{tabular}

${ }^{a}$ Specimens in the Families Chrysomeldidae and Noctuidae are larvae (caterpillars). ${ }^{b}$ Frequency (number of occurrences out of 45 ). 
$\times$ month interaction for invertebrate abundance and richness $(F \geq 3.02$; $\mathrm{df}=6$, $168 ; \mathrm{P} \leq 0.008)$, but not for invertebrate biomass $(\mathrm{F}=1.72 ; \mathrm{df}=6,168 ; \mathrm{P}=$ 0.118) (Figure 1; Table 7).

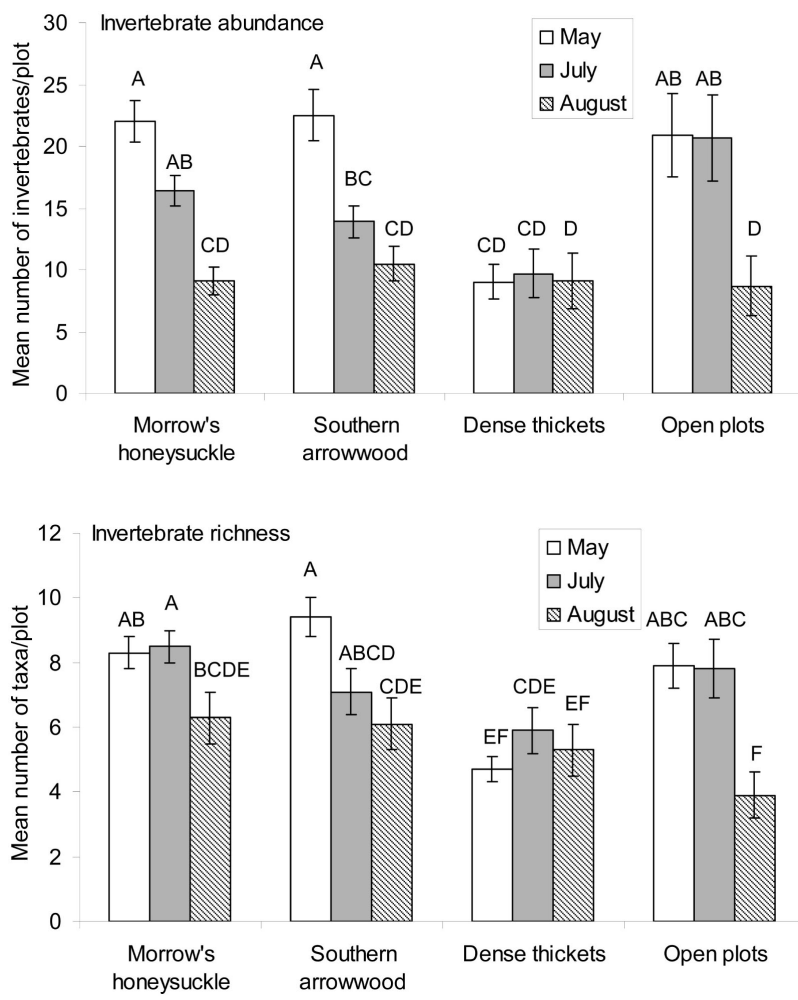

Figure 1. Mean ( $\pm \mathrm{SE})$ ground-dwelling invertebrate abundance (top) and richness (bottom) differed depending on shrub understory type and month at Fort Necessity National Battlefield, Pennsylvania. Means with different letters are significantly different, based on Duncan's multiple range tests $(\mathrm{P}<0.05)$.

Table 7. Mean ( \pm SE) abundance, biomass (dry weight $(\mathrm{mg})$ ), and richness (Family or lowest taxonomic group) of invertebrates ( $\geq 2 \mathrm{~mm}$ in length) sampled in the understory based on shrub type and month at Fort Necessity National Battlefield, Pennsylvania, USA.

\begin{tabular}{cccccc}
\hline Variable & & Abundance & Biomass (mg) & Richness \\
\cline { 5 - 6 } & & $\mathrm{n}$ & $\bar{X} \pm \mathrm{SE}^{*}$ & $\bar{X} \pm \mathrm{SE}$ & $\bar{X} \pm \mathrm{SE}$ \\
\hline Shrub type & & & & \\
Lone Morrow's honeysuckle shrubs & 45 & $15.8 \pm 1.1 \mathrm{~A}$ & $14.5 \pm 2.0 \mathrm{~A}$ & $7.7 \pm 0.4 \mathrm{~A}$ \\
Lone southern arrowwood shrubs & 45 & $15.6 \pm 1.2 \mathrm{~A}$ & $14.5 \pm 1.7 \mathrm{~A}$ & $7.5 \pm 0.5 \mathrm{~A}$ \\
Dense thickets of Morrow's honeysuckle & 45 & $9.2 \pm 1.1 \mathrm{~B}$ & $8.6 \pm 1.8 \mathrm{~B}$ & $5.3 \pm 0.4 \mathrm{~B}$ \\
Open plots with no overstory & 45 & $16.8 \pm 2.0 \mathrm{~A}$ & $12.3 \pm 2.0 \mathrm{AB}$ & $6.5 \pm 0.5 \mathrm{~B}$ \\
Month & & & & \\
May & 60 & $18.6 \pm 1.3 \mathrm{~A}$ & $11.6 \pm 1.1 \mathrm{~A}$ & $7.6 \pm 0.4 \mathrm{~A}$ \\
July & 60 & $15.2 \pm 1.2 \mathrm{~B}$ & $12.7 \pm 1.7 \mathrm{~A}$ & $7.3 \pm 0.4 \mathrm{~A}$ \\
August & 60 & $9.4 \pm 0.9 \mathrm{C}$ & $13.2 \pm 2.0 \mathrm{~A}$ & $5.4 \pm 0.4 \mathrm{~B}$ \\
\hline
\end{tabular}

${ }^{*}$ Means in columns with different letters are significantly different $(\mathrm{P}<0.05)$, based on Duncan's multiple range tests. 


\subsection{Factors Influencing Ground-Dwelling Invertebrates}

For invertebrate abundance in May, the model "shrub" was the best-approximating model (wi $=0.99)$. The remaining models received no empirical support $\left(\triangle \mathrm{AIC}_{\mathrm{c}}\right.$ $\geq 10.14$; wi $\leq 0.01$ ) (Table 2). For invertebrate abundance in July, the model "total herbs" (wi = 0.97) was the best-approximating model, while the remaining six models had no empirical support $\left(\Delta \mathrm{AIC}_{\mathrm{c}} \geq 8.25\right.$; wi $\left.\leq 0.02\right)$ (Table $2)$. In August, the model "total herbs" was the best-approximating model; the models "debris" $\left(\Delta \mathrm{AIC}_{\mathrm{c}}=1.04\right.$; wi $\left.=0.24\right)$ and "native herbs" $\left(\Delta \mathrm{AIC}_{\mathrm{c}}=1.33\right.$; wi $=0.21)$ also received strong empirical support, while the models "soil" $\left(\Delta \mathrm{AIC}_{\mathrm{c}}=3.33\right.$; wi $\left.=0.08\right)$ and "litter" $\left(\Delta \mathrm{AIC}_{\mathrm{c}}=3.84 ;\right.$ wi $\left.=0.06\right)$ received limited support (Table 2).

For invertebrate biomass in May, the model "total herbs" (wi $=0.47$ ) was the best approximating model, though the models "debris" $\left(\Delta \mathrm{AIC}_{\mathrm{c}}=1.85\right.$; wi $=$ $0.19)$, "native herbs" $\left(\Delta \mathrm{AIC}_{\mathrm{c}}=2.51\right.$; wi $\left.=0.13\right)$, and "litter" $\left(\Delta \mathrm{AIC}_{\mathrm{c}}=3.09\right.$; wi $=$ 0.10) also had strong empirical support. The remaining models had little to no support $\left(\Delta \mathrm{AIC}_{\mathrm{c}} \geq 4.37\right.$; wi $\left.\leq 0.05\right)$ (Table 3). In July, the model "total herbs" (wi $=0.81$ ) was the best-approximating model to determine patterns of invertebrate biomass, although the model "debris" $\left(\Delta \mathrm{AIC}_{\mathrm{c}}=3.56\right.$; wi $\left.=0.14\right)$ also had some empirical support; the remaining models received no empirical support $\left(\triangle \mathrm{AIC}_{\mathrm{c}}\right.$ $\geq 7.10$; wi $\leq 0.02$ ) (Table 3). For invertebrate biomass in August, the model "shrub" (wi $=0.47$ ) was the best-approximating model, though the models "native herbs" $\left(\Delta \mathrm{AIC}_{\mathrm{c}}=2.00\right.$, wi $\left.=0.17\right)$, “debris" $\left(\Delta \mathrm{AIC}_{\mathrm{c}}=2.52\right.$, wi $\left.=0.13\right)$, and "total herbs" $\left(\Delta \mathrm{AIC}_{\mathrm{c}}=2.54\right.$, wi $\left.=0.13\right)$ also had strong empirical support. The remaining models had little or no empirical support $\left(\Delta \mathrm{AIC}_{\mathrm{c}} \geq 4.37\right.$, wi $\left.\leq 0.05\right)$. Overall, there was a strong relation among both invertebrate abundance and biomass to total herbaceous cover and shrub type relative to other biotic and abiotic factors (Figure 2; Table 8). Moreover, the proportion of native herbs was

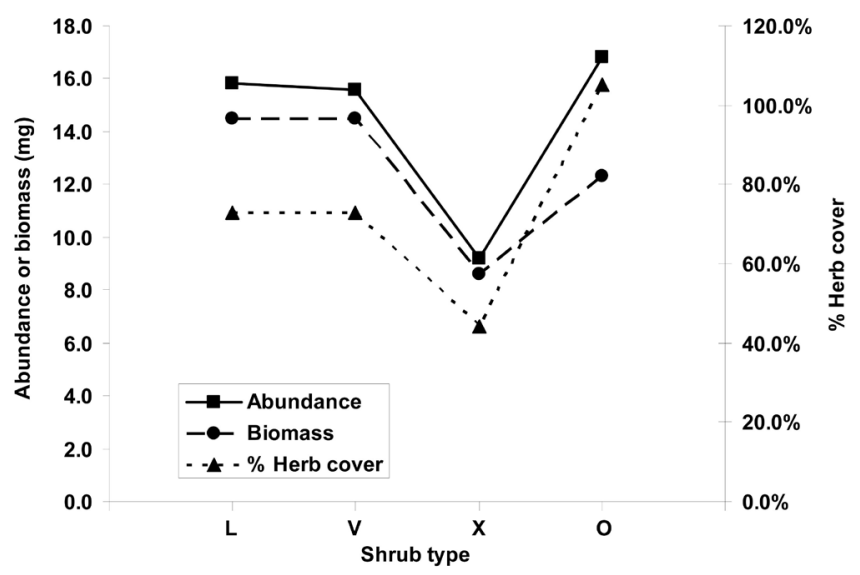

Figure 2. Ground-dwelling mean invertebrate abundance and biomass over all months was positively related to percent herbaceous cover, which in turn was regulated by shrub type ( $\mathrm{L}=$ lone Morrow's honeysuckle; $\mathrm{V}=$ lone southern arrowwood; $\mathrm{X}=$ dense thickets of Morrow's honeysuckle; and $\mathrm{O}=$ open plots with no overstory) within a degraded meadow at Fort Necessity National Battlefield, Pennsylvania, USA. 
Table 8. Mean $( \pm \mathrm{SE})$ values of microhabitat variables recorded under Morrow's honeysuckle shrubs (L), southern arrowwood shrubs (V), dense thickets of Morrow's honeysuckle $(\mathrm{X})$, and open plots with no shrub cover $(\mathrm{O})$ based on month at Fort Necessity National Battlefield, Pennsylvania, USA.

\begin{tabular}{|c|c|c|c|c|}
\hline \multirow{3}{*}{ Microhabitat variables $^{\mathrm{a}}$} & \multicolumn{4}{|c|}{ Shrub type ${ }^{b}$} \\
\hline & $\mathrm{L}$ & $\mathrm{V}$ & $\mathrm{X}$ & $\mathrm{O}$ \\
\hline & $\bar{X} \pm \mathrm{SE}$ & $\bar{X} \pm \mathrm{SE}$ & $\bar{X} \pm \mathrm{SE}$ & $\bar{X} \pm \mathrm{SE}$ \\
\hline \multicolumn{5}{|l|}{ May, $\mathrm{n}=58$} \\
\hline Herb cover $(\%)$ & $50.2 \pm 5.6$ & $49.6 \pm 5.4$ & $34.1 \pm 5.3$ & $75.0 \pm 4.1$ \\
\hline Native cover: total cover (\%) & $48.5 \pm 6.9$ & $64.8 \pm 5.4$ & $39.4 \pm 5.9$ & $61.7 \pm 6.1$ \\
\hline Dry litter wt. (g) & $21.57 \pm 4.27$ & $30.99 \pm 5.35$ & $20.66 \pm 4.84$ & $17.16 \pm 3.01$ \\
\hline Litter N (\%) & $1.30 \pm 0.12$ & $1.49 \pm 0.08$ & $1.48 \pm 0.12$ & $1.22 \pm 0.07$ \\
\hline Soil moisture (\%) & $24.7 \pm 0.8$ & $24.7 \pm 0.7$ & $24.9 \pm 1.1$ & $23.3 \pm 0.7$ \\
\hline Soil temp. $\left({ }^{\circ} \mathrm{C}\right)$ & $15.0 \pm 0.3$ & $16.1 \pm 0.4$ & $14.8 \pm 0.3$ & $16.7 \pm 0.3$ \\
\hline Wet debris wt. (g) & $2.09 \pm 0.45$ & $1.99 \pm 0.22$ & $5.60 \pm 1.08$ & $0.62 \pm 0.09$ \\
\hline \multicolumn{5}{|l|}{ July, $\mathrm{n}=50$} \\
\hline Herb cover $(\%)$ & $99.1 \pm 11.0$ & $106.8 \pm 10.5$ & $50.6 \pm 8.0$ & $155.5 \pm 11.7$ \\
\hline Native cover: total cover (\%) & $64.6 \pm 8.0$ & $68.3 \pm 8.1$ & $56.7 \pm 6.8$ & $72.8 \pm 7.8$ \\
\hline Dry litter wt. (g) & $13.06 \pm 3.99$ & $23.66 \pm 4.46$ & $17.10 \pm 3.37$ & $22.10 \pm 4.73$ \\
\hline Litter N (\%) & $1.07 \pm 0.12$ & $1.28 \pm 0.12$ & $1.23 \pm 0.12$ & $1.11 \pm 0.07$ \\
\hline Soil moisture (\%) & $19.9 \pm 1.14$ & $22.8 \pm 0.8$ & $21.6 \pm 0.7$ & $21.2 \pm 2.3$ \\
\hline Soil temp. $\left({ }^{\circ} \mathrm{C}\right)$ & $21.8 \pm 0.9$ & $20.4 \pm 0.4$ & $20.6 \pm 0.3$ & $22.9 \pm 0.6$ \\
\hline Wet debris wt. (g) & $1.03 \pm 0.16$ & $0.92 \pm 0.19$ & $2.86 \pm 0.52$ & $0.92 \pm 0.16$ \\
\hline \multicolumn{5}{|l|}{ August, $\mathrm{n}=59$} \\
\hline Herb cover $(\%)$ & $61.3 \pm 6.2$ & $64.1 \pm 7.4$ & $46.0 \pm 7.4$ & $88.9 \pm 6.3$ \\
\hline Native cover: total cover $(\%)$ & $75.4 \pm 3.4$ & $77.7 \pm 4.5$ & $58.0 \pm 7.4$ & $81.5 \pm 4.0$ \\
\hline Dry litter wt. (g) & $19.69 \pm 2.76$ & $40.68 \pm 4.92$ & $16.00 \pm 2.94$ & $27.84 \pm 4.71$ \\
\hline Litter N (\%) & $1.27 \pm 0.12$ & $1.53 \pm 0.11$ & $1.45 \pm 0.11$ & $1.13 \pm 0.09$ \\
\hline Soil moisture (\%) & $14.2 \pm 0.5$ & $14.5 \pm 0.3$ & $13.6 \pm 0.4$ & $13.5 \pm 0.6$ \\
\hline Soil temp. $\left({ }^{\circ} \mathrm{C}\right)$ & $25.2 \pm 0.6$ & $25.3 \pm 0.7$ & $24.2 \pm 0.5$ & $26.2 \pm 0.5$ \\
\hline Wet debris wt. (g) & $2.35 \pm 0.27$ & $3.33 \pm 0.55$ & $3.31 \pm 0.51$ & $3.56 \pm 0.50$ \\
\hline
\end{tabular}

${ }^{a}$ Microhabitat variables correspond to model parameters in Table $1 .{ }^{b} \mathrm{~L}=$ single Morrow's honeysuckle shrubs; V = single southern arrowwood shrubs; $\mathrm{X}$ = dense thickets of Morrow's honeysuckle shrubs; and O $=$ open plots with no shrub canopy.

consistently depressed under dense thickets of Morrow's honeysuckle relative to the other shrub types (Table 8).

\subsection{Leaf Herbivory}

Over the course of the three sample periods, we analyzed and assigned ranks to 4465 leaves of Morrow's honeysuckle and 1121 leaves of southern arrowwood. Additionally, we photographed 181 leaves of Morrow's honeysuckle and 308 leaves of southern arrowwood that showed signs of herbivory; 615 leaves of Morrow's honeysuckle and 91 leaves of southern arrowwood were photographed that did not shows signs of leaf herbivory. Mean $( \pm S E)$ total leaf area of Mor- 
row's honeysuckle with leaf herbivory was $3.38 \pm 0.17 \mathrm{~cm}^{2}$, while mean leaf area of leaves that were entire was $3.13 \pm 0.07 \mathrm{~cm}^{2}$. Mean $( \pm$ SE) total leaf area of southern arrowwood leaves with signs of herbivory was $9.77 \pm 0.31 \mathrm{~cm}^{2}$, while mean area of leaves without signs of herbivory was $7.13 \pm 0.48 \mathrm{~cm}^{2}$.

Southern arrowwood was significantly greater than Morrow's honeysuckle in percent of leaves with evidence of herbivory and leaf rank $(\mathrm{F} \geq 139.30$; $\mathrm{df}=1,84$; $\mathrm{P}<0.001)$, as well as leaf area consumed $(\mathrm{F}=18.31$, $\mathrm{df}=1,77 ; \mathrm{P}<0.001)$. Overall, southern arrowwood had $284.3 \mathrm{~cm}^{2}$ of leaf area consumed per $1 \mathrm{~m}^{2}$ of leaf area, while Morrow's honeysuckle had $29.7 \mathrm{~cm}^{2}$ of leaf area consumed per $1 \mathrm{~m}^{2}$ of leaf area. Herbivory metrics in May were consistently lower relative to metrics in July and August. There were significant differences among months for percent of leaves with evidence of herbivory and leaf $\operatorname{rank}(\mathrm{F} \geq 49.61 ; \mathrm{df}=2,84 ; \mathrm{P}<$ $0.001)$, as well as leaf area consumed and percent of leaf consumed ( $F=5.40$; df $=2,77 ; \mathrm{P}=0.006$ ). Moreover, there were significant shrub $\times$ month interaction effects for percent of leaves with evidence of herbivory and leaf rank $(\mathrm{F} \geq 8.89$; $\mathrm{df}$ $=2,84 ; \mathrm{P}<0.001$ ) (Figure 3 ), though there were no significant differences in mean leaf area consumed $(\mathrm{F}=2.90 ; \mathrm{df}=2,77 ; \mathrm{P}=0.061)$ (Table 9).
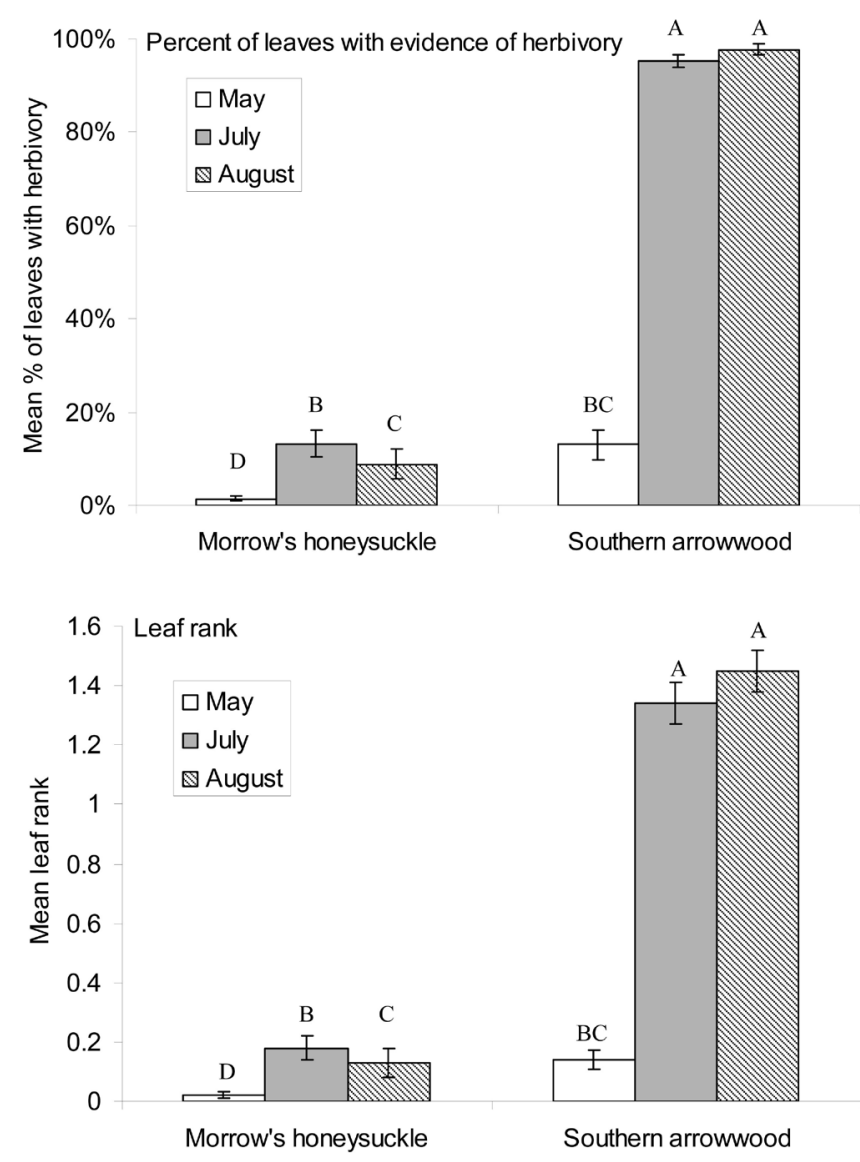

Figure 3. Mean $( \pm$ SE) percent of leaves with evidence of herbivory (top) and leaf rank (bottom; see last sentence in Section 2.4 for rank definitions) differed between shrub type and seasons at Fort Necessity National Battlefield, Pennsylvania. Means with different letters are significantly different, based on Duncan's multiple range tests $(\mathrm{P}<0.05)$. 
Table 9. Morrow's honeysuckle and southern arrowwood mean $( \pm \mathrm{SE})$ percent of leaves with evidence of herbivory, leaf area consumed $\left(\mathrm{cm}^{2}\right)$, and herbivory rank at Fort Necessity National Battlefield, Pennsylvania, USA.

\begin{tabular}{ccccc}
\hline Variable & $\mathrm{n}^{\mathrm{a}}$ & $\begin{array}{c}\text { Percent of leaves } \\
\text { w/evidence of herbivory }\end{array}$ & $\begin{array}{c}\text { Leaf area } \\
\text { consumed }\left(\mathrm{cm}^{2}\right)\end{array}$ & Leaf rank \\
\cline { 3 - 5 } & & $\bar{X} \pm \mathrm{SE}^{\mathrm{b}}$ & $\bar{X} \pm \mathrm{SE}$ & $\bar{X} \pm \mathrm{SE}$ \\
\hline Shrub & & & \\
Morrow's honeysuckle & 45 & $7.9 \pm 1.5 \mathrm{~B}$ & $0.12 \pm 0.03 \mathrm{~B}$ & $0.11 \pm 0.02 \mathrm{~B}$ \\
Southern arrowwood & 45 & $68.6 \pm 6.1 \mathrm{~A}$ & $0.38 \pm 0.07 \mathrm{~A}$ & $0.98 \pm 0.10 \mathrm{~A}$ \\
Month & & & & \\
May & 30 & $7.2 \pm 2.0 \mathrm{~B}$ & $0.17 \pm 0.05 \mathrm{~B}$ & $0.08 \pm 0.02 \mathrm{~B}$ \\
July & 30 & $54.2 \pm 7.8 \mathrm{~A}$ & $0.26 \pm 0.06 \mathrm{~A}$ & $0.76 \pm 0.11 \mathrm{~A}$ \\
August & 30 & $53.3 \pm 8.4 \mathrm{~A}$ & $0.31 \pm 0.08 \mathrm{~A}$ & $0.79 \pm 0.13 \mathrm{~A}$ \\
\hline
\end{tabular}

${ }^{a}$ For leaf area consumed, $n$ is as follows: Morrow's honeysuckle $(n=42)$, southern arrowwood $(n=41)$, May $(n=25)$, July $(n=30)$, August $(n=28)$. ${ }^{b}$ Means in columns with different letters are significantly different $(\mathrm{P}<0.05)$, based on Duncan's multiple range tests.

\section{Discussion}

\subsection{Invertebrates}

While several studies have examined invertebrate abundance on exotic versus native plants [12] [13] [14], this is the first study we are aware of that examined the effects of exotic plants on invertebrate biomass [15]. Since invertebrate biomass is closely linked to the energetic value of an invertebrate food item [78] [79] [80], our findings could have implications for organisms at higher trophic levels, such as songbirds. For instance, we found that within the shrub strata, the native shrub contained lower overall invertebrate biomass than either dense thickets or single shrubs of exotic Morrow's honeysuckle (though there was no such trend when mean biomass was divided by shrub type and month). However, the native shrub contained five times more larval leaf chewer biomass than found in thickets of the exotic shrub and one and one-half times more than found on single Morrow's honeysuckle shrubs. Lower levels of larval leaf chewers could possibly increase foraging distance and time for some species of songbirds [81], particularly during the nesting season when invertebrate protein, especially from larval leaf chewers, makes up a large portion of the diet of nestlings [82]. For example, Prairie Warblers (Dendroica discolor Vieillot) were common nesting songbirds within our study site and primarily feed their young larval leaf chewers such as caterpillars [82], which are higher in nutrients than most other groups of invertebrates [83]. Other studies reveal that birds nesting in Amur honeysuckle have lower rates of nest success than nests found in native shrubs and trees; lower nest height, greater shrub volume, lack of sharp thorns, and branch architecture that facilitate movement of predators are thought to contribute to higher rates of nest predation in these exotic shrubs relative to native shrubs and trees [31] [32]. If these nests are in a matrix of exotic shrubs which 
produce few larval leaf chewers, then the extra time and effort spent foraging for preferred prey could also be a contributing factor leading to increased rates of nest predation. Clearly more research is needed to ascertain whether there is a link between reduced biomass of important invertebrate prey items on exotic bush honeysuckles and bird foraging behavior and subsequent nest success.

Invertebrate composition within the shrub strata differed among the three shrub types. Ants (Hymenoptera: Formicidae) were five times more abundant on southern arrowwood relative to lone shrubs of Morrow's honeysuckle and nearly 14 times more abundant relative to dense thickets of the exotic shrub. We attribute this pattern to large numbers of aphids (Homoptera: Aphididae) feeding on the native shrub. Ants feed on honeydew produced by aphids and protect aphid colonies from predators and sooty mold contamination [84] [85]; this strong correlation between aphid and ant abundance has been observed before (e.g. [83]). Moreover, we also observed ants feeding directly from sugar exudates arising from immature berries of southern arrowwood, even in the absence of aphids. Lady beetles (Coleoptera: Coccinellidae) had the greatest biomass on southern arrowwood, where they were the top invertebrate in terms of biomass. We also attribute this pattern to the presence of aphids feeding on southern arrowwood; the beetles were observed feeding on the aphids, a favorite prey item for ladybugs [64].

Invertebrate biomass was consistently reduced in the understory below dense thickets of Morrow's honeysuckle over all months. In a Kentucky forest, northern slimy salamanders (Plethodon glutinosus Green) and green frogs (Rana clamitans Latreille) found in areas with a dense cover of Amur honeysuckle had lower body mass compared to non-invaded areas, suggesting that the shrub might be reducing the availability of prey items, although no quantitative data on invertebrate availability was obtained [33]. Invertebrate richness also was significantly lower under dense thickets of Morrow's honeysuckle, a trend also found with spiders found in the understory of hedges dominated by Amur honeysuckle [30].

\subsection{Factors Influencing Invertebrate Patterns}

Overall, patterns of invertebrate abundance and biomass in the understory were driven by percent cover of herbs, which in turn was ultimately influenced by the type of shrub overstory. Reduced herbaceous cover in areas dominated by Amur honeysuckle was thought to be the proximate factor responsible for depressing spider richness [30] and amphibian condition and diversity [33]. Other studies also have documented the positive correlation of understory herbaceous cover and invertebrate abundance [86]-[91] but not all studies support this [92]. The reduced abundance, biomass, and richness under dense thickets of Morrow's honeysuckle is not surprising given the numerous studies showing the shrubs' impact on herbaceous diversity and cover [4] [26] [27]. It is interesting to note that the proportion of native herbaceous species was reduced under dense thickets of Morrow's honeysuckle relative to the other shrub types; future studies 
should assess whether this trend occurs with other bush honeysuckle species.

Our study revealed that patterns of invertebrate abundance are not necessarily correlated with invertebrate biomass. For instance, invertebrates captured in the shrub strata of southern arrowwood were greater in abundance than the other two shrub types but had significantly less biomass compared to the other shrub types. Moreover, there were few invertebrate families that were among both the five most abundant invertebrate groups and five heaviest groups in terms of total biomass. We caution researchers that correlating invertebrate abundance with biomass may be misleading.

One limitation of our study is that we did not identify invertebrates to species. There is a possibility that exotic invertebrates may have contributed a significant portion of the overall invertebrate abundance, biomass, and richness and may have skewed our expected results (i.e. enemy release hypothesis-significantly greater abundance, biomass, and richness of invertebrates found in the shrub strata of the native shrub relative to the exotic shrub). For instance, honey bees (Apis mellifera L, Hymenoptera: Apidae) were sampled during May on Morrow's honeysuckle when the shrubs were flowering. We only captured three of these insects on single shrubs of Morrow's honeysuckle, but because of their large size relative to other invertebrates, they had the second most total mass of invertebrate groups found on this shrub type. We also captured one Japanese beetle (Popillia japonica, Coleoptera: Scarabaeidae) on southern arrowwood. It is possible that more species of exotic invertebrates were sampled. For example, in a simultaneous experiment at our study site researching patterns of earthworm abundance, biomass, and richness, only four species of earthworms were sampled and all four species were exotic [93]. However, based on the limited herbivory on Morrow's honeysuckle relative to southern arrowwood, we believe that if any exotic phytophagous invertebrates were present that fed on Morrow's honeysuckle, we would have observed more leaf damage.

It is also possible that native Lonicera-specific herbivores may have been feeding on Morrow's honeysuckle. Related species of plants often present similar chemical cues that attract herbivores [94]. Introduced plants that are closely related to native plants often draw the same suite of herbivores and have similar rates of herbivory [95] [96]. However, native bush honeysuckles are rare in Pennsylvania, making it doubtful that a host shift to Morrow's honeysuckle has occurred. Three of the four native species, hairy honeysuckle (L. hirsuta Eat.), swamp fly honeysuckle (L. oblongifolia (Goldie) Hook.), and mountain fly honeysuckle (L. villosa (Michx.) J. A. Schultes) have a state heritage rank of S1 $(<5$ populations recorded in the state) [97] [98]. While not ranked in Pennsylvania, a fourth native honeysuckle, limber honeysuckle ( $L$. dioica L.) has a rank of S3 (21 - 100 occurrences in the state) in neighboring West Virginia and is infrequently encountered [98]. Moreover, no native bush honeysuckles have been recorded at Fort Necessity National Battlefield and the relatively low rates of herbivory that we documented support our belief that few, if any, native Lonicera-specific hosts feed on Morrow's honeysuckle. 
Because the native shrubs that we sampled occurred in a landscape matrix dominated by the exotic Morrow's honeysuckle, there is a possibility that the exotic shrubs impeded herbivores from moving to these patches of native shrubs (i.e. fragmentation), thereby reducing the overall abundance, biomass, and richness of invertebrates found on the native shrubs. For example, a planthopper (Homoptera: Cicadellidae) and its specialist parasitoid (Hymenoptera: Mymaridae) were $50 \%$ lower in native patches of the host plant that were embedded in a matrix of exotic grass compared to a matrix dominated by the native host plant [99]. Other studies reveal that the composition of the habitat between host-patch patches can significantly affect interpatch movement rates of herbivores [100] [101] [102]. Our results may have differed if the native shrubs we sampled were located in native vegetative communities, though we can only speculate since we did not measure landscape effects.

\subsection{Leaf Herbivory}

Leaf herbivory was nearly 10 times more on the native southern arrowwood than on Morrow's honeysuckle. Herbarium specimens of Amur honeysuckle had less leaf damage compared to native shrubs, suggesting that the shrub may be relatively free from herbivores and/or pathogens; the lack of herbivores and pathogens may be partly responsible for its success in invading foreign soils [41]. Branches that were newly formed on Morrow's honeysuckle typically had larger leaves than older branches (personal observation). Since insects often prefer newer, more palatable leaves [12], we believe the larger size of leaves on younger branches explains the reason why larger leaves were more likely to have herbivory. On southern arrowwood, we believe the larger leaves were older and thereby had a greater chance of being exploited by herbivores. In our samples, we observed shrubs affected by the fungus Insolibasidium deformans C. J. Gould (Auriculariaceae) (fungus id confirmed by W. MacDonald 2004, West Virginia University, Morgantown), a blight specific to Lonicera that causes a crinkling and browning of the leaves [103]. However, we did not quantitatively measure this disease and it did not seem to have a deleterious impact on the shrub. Overall our results suggest that the enemy release hypothesis is at least partly responsible for the success of Morrow's honeysuckle invading and persisting at our study site.

\subsection{Conclusion}

Many land managers believe that bush honeysuckles, including Morrow's honeysuckle, create sufficient habitat for songbirds and game species [104]. Moreover, the abundant, conspicuous red berries are often cited as being beneficial for wildlife, particularly songbirds [35] [105] [106]. However, in light of recent studies showing increased rates of nest predation in bush honeysuckles [31] [32] and evidence of deleterious effects of the shrubs on amphibians [33], coupled with our findings of decreased larval leaf chewer biomass in the shrub strata of the exotic shrub and significantly lower invertebrate biomass under dense thick- 
ets of the shrub, we believe that areas dominated by Morrow's honeysuckle may be at best, marginal bird habitat, and at worst, ecological traps.

\section{Acknowledgements}

We thank J. Edalgo, J. E. Love, M. Hepner, B. Crokus, J. Alexander, V. Wells, E. Ralph, D. Ralph, A. Strippel, and A. Williams for field and lab assistance. V. Kondo, L. Butler, R. Utz, and D. Hartman verified invertebrate specimens. W. Grafton aided in the identification of plant specimens. L. Butler gave helpful advice on the art of invertebrate sampling. A. B. Billings provided statistical advice. J. McGraw and the late C. Ranson reviewed earlier drafts of this manuscript. We thank the staff of Fort Necessity National Battlefield, especially C. Ranson, for her support and advice throughout the project. Funding for this project was provided by the National Park Service and Fort Necessity National Battlefield. J. T. Anderson was supported by the National Science Foundation (01A-1458952) and the National Institute of Food and Agriculture (McStennis Project WVA00117 and WVA00812) during manuscript preparation.

\section{Conflicts of Interest}

The authors declare no conflicts of interest regarding the publication of this paper.

\section{References}

[1] Mack, R.N. and Lonsdale, W.M. (2001) Humans as Global Plant Dispersers: Getting More than We Bargained for. BioScience, 51, 95-102. https://doi.org/10.1641/0006-3568(2001)051[0095:HAGPDG]2.0.CO;2

[2] Pimentel, D., Lach, L., Zuniga, R. and Morrison, D. (2000) Environmental and Economic Costs of Nonindigenous Species in the United States. BioScience, 50, 53-65. https://doi.org/10.1641/0006-3568(2000)050[0053:EAECON]2.3.CO;2

[3] Slobodchikoff, C.N. and Doyen, J.T. (1977) Effects of Ammophila arenaria on Sand Dune Arthropod Communities. Ecology, 58, 1171-1175. https://doi.org/10.2307/1936939

[4] Collier, M.H., Vankat, J.L. and Hughes, M.R. (2002) Diminished Plant Richness and Abundance below Lonicera maackii, an Invasive Shrub. American Midland Naturalist, 147, 60-71. https://doi.org/10.1674/0003-0031(2002)147[0060:DPRAAB]2.0.CO;2

[5] Vitousek, P.M., Walker, L.R., Whiteaker, L.D., Mueller-Dombois, D. and Matson, P.A. (1987) Biological Invasion by Myrica faya Alters Ecosystem Development in Hawaii. Science, 238, 802-804. https://doi.org/10.1126/science.238.4828.802

[6] Rejmánek, M. and Richardson, D.M. (1996) What Attributes Make Some Plant Species More Invasive? Ecology, 77, 1655-1661. https://doi.org/10.2307/2265768

[7] Callaway, R.M. and Aschehoug, E.T. (2000) Invasive Plants versus Their New and Old Neighbors: A Mechanism for Exotic Invasion. Science, 290, 521-523. https://doi.org/10.1126/science.290.5491.521

[8] Levine, J.M. (2000) Species Diversity and Biological Invasions: Relating Local Process to Community Pattern. Science, 288, 852-854. 
https://doi.org/10.1126/science.288.5467.852

[9] Elton, C.S. (1958) The Ecology of Invasion by Plants and Animals. Chapman and Hall, London. https://doi.org/10.1007/978-1-4899-7214-9

[10] Lodge, D.M. (1993) Biological Invasions: Lessons for Ecology. Trends in Ecology \& Evolution, 8, 133-137. https://doi.org/10.1016/0169-5347(93)90025-K

[11] Tilman, D. (1999) The Ecological Consequences of Changes in Biodiversity: A Search for General Principles. Ecology, 80, 1455-1474. https://doi.org/10.2307/176540

[12] Strong, D.R., Lawton, J.H. and Southwood, T.R.E. (1984) Insects on Plants. Harvard University Press, Cambridge.

[13] Schierenbeck, K.A., Mack, R.N. and Sharitz, R.R. (1994) Effects of Herbivory on Growth and Biomass Allocation in Native and Introduced Species of Lonicera. Ecology, 75, 1661-1672. https://doi.org/10.2307/1939626

[14] Yela, J.L. and Lawton, J.H. (1997) Insect Herbivore Loads on Native and Introduced Plants: A Preliminary Study. Entomologia Experimentalis et Applicata, 85, 275-279. https://doi.org/10.1046/j.1570-7458.1997.00258.x

[15] Tallamy, D.M. (2004) Do Alien Plants Reduce Insect Biomass? Conservation Biology, 18, 1689-1692. https://doi.org/10.1111/j.1523-1739.2004.00512.x

[16] Dickinson, M.B. (1999) Field Guide to the Birds of North America. 3rd Edition, National Geographic Society, Washington DC.

[17] Burke, D.M. and Nol, E. (1998) Influence of Food Abundance, Nest-Site Habitat, and Forest Fragmentation on Breeding Ovenbirds. Auk, 115, 96-104. https://doi.org/10.2307/4089115

[18] Marra, P.P., Hobson, K.A. and Holmes, R.T. (1998) Linking Winter and Summer Events in a Migratory Bird Using Stable-Carbon Isotopes. Science, 282, 1884-1886. https://doi.org/10.1126/science.282.5395.1884

[19] Zanette, L., Doyle, P. and Trémont, S.M. (2000) Food Shortage in Small Fragments: Evidence from an Area-Sensitive Passerine. Ecology, 81, 1654-1666. https://doi.org/10.1890/0012-9658(2000)081[1654:FSISFE]2.0.CO;2

[20] Johnson, M.D. and Sherry, T.W. (2001) Effects of Food Availability on the Distribution of Migratory Warblers among Habitats in Jamaica. Journal of Animal Ecology, 70, 546-560. https://doi.org/10.1046/j.1365-2656.2001.00522.x

[21] Folse, L.J. (1982) An Analysis of Avifauna-Resource Relationships on the Serengeti Plains. Ecological Monographs, 52, 111-127. https://doi.org/10.2307/1942607

[22] Rodewald, A.D. and Vitz, A.C. (2005) Edge- and Area-Sensitivity of Shrubland Birds. The Journal of Wildlife Management, 69, 681-688. https://doi.org/10.2193/0022-541X(2005)069[0681:EAAOSB]2.0.CO;2

[23] Batcher, M.S. and Stiles, S.A. (2000) Element Stewardship Abstract for Lonicera maackii (Rupr.) Maxim (Amur Honeysuckle), Lonicera morrowii A. Gray (Morrow's Honeysuckle), Lonicera tatarica L. (Tatarian Honeysuckle), Lonicera $\times$ bella Zabel (Bell's Honeysuckle). The Nature Conservancy, Arlington. https://www.invasive.org/gist/esadocs/documnts/loni_sp.pdf

[24] Rehder, A. (1940) A Manual of Cultivated Trees and Shrubs. 2nd Edition, MacMillan Publishing Company, New York.

[25] Luken, J.O. and Thieret, J.W. (1995) Amur Honeysuckle (Lonicera maackii, Caprifoliaceae): Its Ascent, Decline, and Fall. Sida, 16, 479-503.

[26] Woods, K.D. (1993) Effects of Invasion by Lonicera tatarica L. on Herbs and Tree 
Seedlings in Four New England Forests. American Midland Naturalist, 130, 62-74. https://doi.org/10.2307/2426275

[27] Hartman, K.M. and McCarthy, B.C. (2004) Restoration of a Forest Understory after the Removal of an Invasive Shrub, Amur Honeysuckle (Lonicera maackii). Restoration Ecology, 12, 154-165. https://doi.org/10.1111/j.1061-2971.2004.00368.x

[28] Miller, K.E. and Gorchov, D.L. (2004) The Invasive Shrub, Lonicera maackii, Reduces Growth and Fecundity of Perennial Forest Herbs. Oecologia, 139, 359-375. https://doi.org/10.1007/s00442-004-1518-2

[29] Gorchov, D.L. and Trisel, D.E. (2003) Competitive Effects of the Invasive Shrub, Lonicera maackii (Rupr.) Herder (Caprifoliaceae), on the Growth and Survival of Native Tree Seedlings. Plant Ecology, 166, 13-24.

[30] Buddle, C.M., Higgins, S. and Rypstra, A.L. (2004) Ground-Dwelling Spider Assemblages Inhabiting Riparian Forests and Hedgerows in an Agricultural Landscape. American Midland Naturalist, 151, 15-26. https://doi.org/10.1674/0003-0031(2004)151[0015:GSAIRF]2.0.CO;2

[31] Schmidt, K.A. and Whelan, C.J. (1999) Effects of Exotic Lonicera and Rhamnus on Songbird Nest Predation. Conservation Biology, 13, 1502-1506. https://doi.org/10.1046/j.1523-1739.1999.99050.x

[32] Borgmann, K.L. and Rodewald, A.D. (2004) Nest Predation in an Urbanizing Landscape: The Role of Exotic Shrubs. Ecological Applications, 14, 1757-1765. https://doi.org/10.1890/03-5129

[33] McEvoy, N.L. and Durtsche, R.D. (2004) Effect of the Invasive Shrub Lonicera maackii (Caprifoliaceae; Amur Honeysuckle) on Autumn Herptofauna Biodiversity. Journal of the Kentucky Academy of Science, 65, 27-32.

[34] Luken, J.O. and Goessling, N. (1995) Seedling Distribution and Potential Persistence of the Exotic Shrub Lonicera maackii in Fragmented Forests. American Midland Naturalist, 133, 124-130. https://doi.org/10.2307/2426353

[35] Ingold, J.L. and Craycraft, M.J. (1983) Avian Frugivory on Honeysuckle (Lonicera) in Southwestern Ohio in the Fall. Ohio Journal of Science, 83, 256-258.

[36] White, D.W. and Stiles, E.W. (1992) Bird Dispersal of Fruits in Species Introduced into Eastern North America. Canadian Journal of Botany, 70, 1689-1696. https://doi.org/10.1139/b92-208

[37] Vellend, M. (2002) A Pest and an Invader: White-Tailed Deer (Odocoileus virginianus Zimm.) as a Seed Dispersal Agent for Honeysuckle Shrubs (Lonicera L.). Natural Areas Journal, 22, 230-234.

[38] Barnes, W.J. and Cottam, G. (1974) Some Autoecological Studies of the Lonicera $\times$ bella Complex. Ecology, 55, 40-50. https://doi.org/10.2307/1934616

[39] Luken, J.O. (1988) Population Structure and Biomass Allocation of the Naturalized Shrub Lonicera maackii (Rupr.) Maxim. in Forest and Open Habitats. American Midland Naturalist, 119, 258-267. https://doi.org/10.2307/2425809

[40] Harrington, R.A., Brown, B.J. and Reich, P.B. (1989) Ecophysiology of Exotic and Native Shrubs in Southern Wisconsin: Relationship of Leaf Characteristics, Resource Availability, and Phenology to Seasonal Patterns of Carbon Gain. Oecologia, 80, 356-367. https://doi.org/10.1007/BF00379037

[41] Trisel, D.E. and Gorchov, D.L. (1994) Regional Distribution, Ecological Impact, and Leaf Phenology of the Invasive Shrub, Lonicera maackii. The Bulletin of the Ecological Society of America, 75, 231-232.

[42] Love, J.P. (2006) Effects of Morrow's Honeysuckle Control and the Impact of the 
Shrub on Invertebrates at Fort Necessity National Battlefield, Pennsylvania. M.S. Thesis, West Virginia University, Morgantown. https://researchrepository.wvu.edu/etd/2362/

[43] Barnes, W.J. (1972) The Autoecology of the Lonicera $\times$ bella Complex. PhD Dissertation, University of Wisconsin, Madison.

[44] Trisel, D.E. (1997) The Invasive Shrub, Lonicera maackii (Rupr.) Herder (Caprifoliaceae): Factors Contributing to Its Success and Its Effect on Native Species. Ph.D. Dissertation, Miami University, Oxford.

[45] Mahr, D.L. and Dittl, T.G. (1986) Chemical, Natural, and Cultural Control of Hyadaphis tataricae (Homoptera: Aphididae) on Honeysuckle. The Great Lakes Entomologist, 19, 91-100.

[46] Herman, D.E. and Davidson, C.G. (1997) Evaluation of Lonicera taxa for Honeysuckle Aphid Susceptibility, Winter Hardiness and Use. Journal of Environmental Horticulture, 15, 177-182.

[47] Parmenter, R.B., MacMahon, J.A. and Gilbert, C.A.B. (1991) Early Successional Patterns of Arthropod Recolonization on Reclaimed Wyoming Strip Mines: The Grasshoppers (Orthoptera: Gryllacrididae, Tettigoniidae). Environmental Entomology, 20, 135-142. https://doi.org/10.1093/ee/20.1.135

[48] Chapin, F.S., Schulze, E.D. and Mooney, H.A. (1992) Biodiversity and Ecosystem Process. Trends in Ecology \& Evolution, 7, 432-433. https://doi.org/10.1016/0169-5347(92)90141-W

[49] Williams, K.S. (1993) Use of Terrestrial Arthropods to Evaluate Restored Riparian Woodlands. Restoration Ecology, 1, 107-117. https://doi.org/10.1111/j.1526-100X.1993.tb00016.x

[50] Webb, C.E., Oliver, I. and Pik, A.J. (2000) Does Coastal Foredune Stabilization with Ammophila arenaria Restore Plant and Arthropod Communities in Southeastern Australia? Restoration Ecology, 8, 283-288. https://doi.org/10.1046/j.1526-100x.2000.80040.x

[51] Ries, L., Debinski, D.M. and Wieland, M.L. (2001) Conservation Value of Roadside Prairie Restoration to Butterfly Communities. Conservation Biology, 15, 401-411. https://doi.org/10.1046/j.1523-1739.2001.015002401.x

[52] Murdoch, W.W., Evan, F.C. and Peterson, C.H. (1972) Diversity and Pattern in Plants and Insects. Ecology, 53, 819-829. https://doi.org/10.2307/1934297

[53] Antvogel, H. and Bonn, A. (2001) Environmental Parameters and Microspatial Distribution of Insects: A Case Study of Carabids in an Alluvial Forest. Ecography, 24, 470-482. https://doi.org/10.1034/j.1600-0587.2001.d01-203.x

[54] Wang, C., Strazanac, J.S. and Butler, L. (2001) Association between Ants (Hymenoptera: Formicidae) and Habitat Characteristics in Oak-Dominated Forests. Environmental Entomology, 30, 842-848. https://doi.org/10.1603/0046-225X-30.5.842

[55] Hertl, P.T. and Brandenburg, R.L. (2002) Effect of Soil Moisture and Time of Year on Mole Cricket (Orthoptera: Gryllotalpidae) Surface Tunneling. Environmental Entomology, 31, 476-481. https://doi.org/10.1603/0046-225X-31.3.476

[56] Collett, N. (2003) Short and Long-Term Effects of Prescribed Fires in Autumn and Spring on Surface Active Arthopods in Sclerophyll Eucalypt Forests of Victoria. Forest Ecology and Management, 182, 117-138. https://doi.org/10.1016/S0378-1127(03)00009-4

[57] Badejo, M.A., Nathaniel, T.I. and Tian, G. (1998) Abundance of Springtails (Collembola) under Four Agroforestry Tree Species with Contrasting Litter Quality. Bi- 
ology and Fertility of Soils, 27, 15-20. https://doi.org/10.1007/s003740050393

[58] Lindsay, E.A. and French, K. (2006) The Impact of the Weed Chrysanthemoides monilifera spp. Rotundata on Coastal Leaf Litter Invertebrates. Biological Invasions, 8, 177-192. https://doi.org/10.1007/s10530-004-5856-0

[59] Kelso, G.K. (1994) Palynology in Historical Rural Landscape Studies: The Pre-Clearance Forest Border at Great Meadows, Pennsylvania. National Park Service, Tech. Rep. NPS/MARFONE/NRTR 95/067.

[60] Kopas, F.A. (1973) Soil Survey of Fayette County, Pennsylvania. U.S. Department of Agriculture, Soil Conservation Service, U.S. Government Printing Office, Washington DC.

[61] Osborne, K.H. and Allen, W.W. (1999) Allen-vac: An Internal Collection Bag Retainer Allows for Snag-Free Arthropod Sampling in Woody Scrub. Environmental Entomology, 28, 594-596. https://doi.org/10.1093/ee/28.4.594

[62] Buffington, M.L. and Redak, R.A. (1998) A Comparison of Vacuum Sampling versus Sweep-Netting for Arthropod Biodiversity Measurements in California Coastal Sage Scrub. Journal of Insect Conservation, 2, 99-106. https://doi.org/10.1023/A:1009653021706

[63] Burger, J.C., Redak, R.A., Allen, E.B., Rotenberry, J.T. and Allen, M.F. (2003) Restoring Arthropod Communities in Coastal Sage Scrub. Conservation Biology, 17, 460-467. https://doi.org/10.1046/j.1523-1739.2003.01325.x

[64] Bland, R.G. and Jacques, H.E. (1978) How to Know the Insects. 3rd Edition, WCB/McGraw-Hill, Dubuque.

[65] Borror, D.J., Triplehorn, C.A. and Johnson, N.F. (1989) An Introduction to the Study of Insects. 6th Edition, Thomson Learning, Inc., Victoria.

[66] Rogers, L.E., Buschbom, R.L. and Watson, C.R. (1977) Length-Weight Relationships of Shrub-Steppe Invertebrates. Annals of the Entomological Society of America, 70, 51-53. https://doi.org/10.1093/aesa/70.1.51

[67] Schoener, T.W. (1980) Length-Weight Regressions in Tropical and Temperate Forest-Understory Insects. Annals of the Entomological Society of America, 73, 106-109. https://doi.org/10.1093/aesa/73.1.106

[68] Collins, P.T. (1992) Length-Biomass Relationships for Terrestrial Gastropoda and Oligochaeta. American Midland Naturalist, 128, 404-406. https://doi.org/10.2307/2426474

[69] Sample, B.E., Cooper, R.J., Greer, R.D. and Whitmore, R.C. (1993a) Estimation of Insect Biomass by Length and Width. American Midland Naturalist, 129, 234-240. https://doi.org/10.2307/2426503

[70] Hódar, J.A. (1996) The Use of Regression Equations for Estimation of Arthropod Biomass in Ecological Studies. Acta Oecologica, 17, 421-433.

[71] Benke, A.C., Huryn, A.D., Smock, L.A. and Wallace, J.B. (1999) Length-Mass Relationships for Freshwater Macroinvertebrates in North America with Particular Reference to the Southeastern United States. Journal of the North American Benthological Society, 18, 308-343. https://doi.org/10.2307/1468447

[72] Sabo, J.L., Bastow, J.L. and Power, M.E. (2002) Length-Mass Relationships for Adult Aquatic and Terrestrial Invertebrates in a California Watershed. Journal of the North American Benthological Society, 21, 336-343. https://doi.org/10.2307/1468420

[73] Kartesz, J.T. (1999) Synthesis of the North American Flora. Version 1.0. North Carolina Botanical Garden, Chapel Hill. 
[74] Hawk, P.B., Oser, B.L. and Summerson, W.H. (1947) Practical Physiological Chemistry. 12th Edition, Blakiston, Toronto.

[75] Rasband, W.S. (2005) ImageJ. U.S. National Institute of Health, Bethesda. http://rsb.info.nih.gov/ij

[76] Burnham, K.P. and Anderson, D.R. (2002) Model Selection and Multimodel Inference: A Practical Information-Theoretic Approach. 2nd Edition, Springer-Verlag, New York.

[77] Conover, W.J. and Iman, R.L. (1981) Rank Transformation as a Bridge between Parametric and Nonparametric Statistics. The American Statistician, 35, 124-129. https://doi.org/10.2307/2683975

[78] Krebs, J.R. and McCleery, R.H. (1984) Optimization in Behavioral Ecology. In: Krebs, J.R., Ed., Behavioral Ecology. An Evolutionary Approach, Blackwell Scientific, Oxford, 91-121.

[79] Karasov, W.H. (1990) Digestion in Birds: Chemical and Physiological Determinants and Ecological Applications. Studies in Avian Biology, 13, 391-415.

[80] Johnson, M.D. (2000) Evaluation of an Arthropod Sampling Technique for Measuring Food Availability for Forest Insectivorous Birds. Journal of Field Ornithology, 71, 88-109. https://doi.org/10.1648/0273-8570-71.1.88

[81] Sample, B.E., Cooper, R.J. and Whitmore, R.C. (1993) Dietary Shifts among Songbirds from a Diflubenzuron-Treated Forest. Condor, 95, 616-624. https://doi.org/10.2307/1369605

[82] Nolan Jr., V., Ketterson, E.D. and Buerkle, C.A. (2020) Prairie Warbler (Setophaga discolor), Version 1.0. In: Poole, A.F., Ed., Birds of the World, Cornell Lab of Ornithology, Ithaca, NY, USA. https://doi.org/10.2173/bow.prawar.01

[83] Schowalter, T.D., Webb, J.W. and Crossley, Jr., D.A. (1981) Community Structure and Nutrient Content of Canopy Arthropods in Clearcut and Uncut Forest Ecosystems. Ecology, 62, 1010-1019. https://doi.org/10.2307/1937000

[84] Way, M.J. (1963) Mutualism between Ants and Honeydew-Producing Homoptera. Annual Review of Entomology, 8, 307-344. https://doi.org/10.1146/annurev.en.08.010163.001515

[85] Petal, J. (1978) The Role of Ants in Ecosystems. In: Brian, M.V., Ed., Production Ecology of Ants and Termites, Cambridge University Press, Cambridge, 293-325.

[86] Webb, N.R., Clarke, R.T. and Nicholas, J.T. (1984) Invertebrate Diversity on Fragmented Calluna-Heathland: Effects of Surrounding Vegetation. Journal of Biogeography, 11, 41-46. https://doi.org/10.2307/2844774

[87] Hendrix, S.D., Brown, V.K. and Dingle, H. (1988) Arthropod Guild Structure during Early Old Field Succession in a New and Old World Site. Journal of Animal Ecology, 57, 1053-1065. https://doi.org/10.2307/5111

[88] Samways, M.J., Caldwell, P.M. and Osborn, R. (1996) Ground-Living Invertebrate Assemblages in Native, Planted and Invasive Vegetation in South Africa. Agriculture, Ecosystems \& Environment, 59, 19-32. https://doi.org/10.1016/0167-8809(96)01047-X

[89] Haddad, N.M., Tilman, D., Haarstad, J., Ritchie, M. and Knops, J.M.H. (2001) Contrasting Effects of Plant Richness and Composition on Insect Communities: A Field Experiment. The American Naturalist, 58, 17-35. https://doi.org/10.1086/320866

[90] Jamison, B.E., Robel, R.J., Pontius, J.S. and Applegate, R.D. (2002) Invertebrate Biomass: Associations with Lesser Prairie-Chicken Habitat Use and Sand Sagebrush Density in Southwestern Kansas. Wildlife Society Bulletin, 30, 517-526. 
[91] Allombert, S., Stockton, S. and Martin, J. (2005) A Natural Experiment on the Impact of Overabundant Deer on Forest Invertebrates. Conservation Biology, 19 , 1917-1929. https://doi.org/10.1111/j.1523-1739.2005.00280.x

[92] Steenkamp, H.E. and Chown, S.L. (1996) Influence of Dense Stands of an Exotic Tree, Prosopis glandulosa Benson, on a Savanna Dung Beetle (Coleoptera: Scarabaeinae) Assemblage in South Africa. Biological Conservation, 78, 305-311. https://doi.org/10.1016/S0006-3207(96)00047-X

[93] Edalgo, J.A. and Anderson J.T. (2009) Predicting Factors Impacting Earthworms (Annelida: Lumbricidae) in a Degraded Pennsylvania Ecosystem. Proceedings of the West Virginia Academy of Sciences, 81, 1-14. http://pwvas.org/index.php/pwvas/issue/viewIssue/95/85

[94] Ehrlich, P.R. and Raven, P.H. (1964) Butterflies and Plants: A Study in Coevolution. Evolution, 18, 568-608. https://doi.org/10.1111/j.1558-5646.1964.tb01674.x

[95] Connor, E.F., Faeth, S.H., Simberloff, D. and Opler, P.A. (1980) Taxonomic Isolation and the Accumulation of Herbivorous Insects: A Comparison of Introduced and Native Trees. Ecological Entomology, 5, 205-211. https://doi.org/10.1111/j.1365-2311.1980.tb01143.x

[96] Agrawal, A.A. and Kotanen, P.M. (2003) Herbivores and the Success of Exotic Plants: A Phylogenetically Controlled Experiment. Ecology Letters, 6, 712-715. https://doi.org/10.1046/j.1461-0248.2003.00498.x

[97] Rhoads, A.F. and Klein, Jr., W.K. (1993) The Vascular Flora of Pennsylvania: Annotated Checklist and Atlas. American Philosophical Society, Philadelphia.

[98] NatureServe (2005) NatureServe Explorer: An Online Encyclopedia of Life [Web Application]. Version 4.6. NatureServe, Arlington. http://www.natureserve.org/explorer

[99] Cronin, J.T. and Haynes, K.J. (2004) An Invasive Plant Promotes Unstable Host-Parasitoid Patch Dynamics. Ecology, 85, 2772-2782. https://doi.org/10.1890/04-0303

[100] Roland, J., Keyghobadi, N. and Fownes, S. (2000) Alpine Parnassius Butterfly Dispersal: Effects of Landscape and Population Size. Ecology, 81, 1642-1653. https://doi.org/10.1890/0012-9658(2000)081[1642:APBDEO]2.0.CO;2

[101] Rickets, T.H. (2001) The Matrix Matters: Effective Isolation in Fragmented Landscapes. The American Naturalist, 158, 87-99. https://doi.org/10.1086/320863

[102] Haynes, K.J. and Cronin, J.T. (2003) Matrix Composition Affects the Spatial Ecology of a Prairie Planthopper. Ecology, 84, 2856-2866. https://doi.org/10.1890/02-0611

[103] Sinclair, W.A., Lyon, H.H. and Johnson, W.T. (1987) Diseases of Shrubs and Trees. Comstock Publishing Associates, Cornell University Press, Ithaca.

[104] VanDruff, L.W., Bolen, E.G. and San Julian, G.J. (1996) Management of Urban Wildlife. In: Bookhout, T.A., Ed., Research and Management Techniques for Wildlife and Habitats, 5th Edition, The Wildlife Society, Allen Press, Inc., Lawrence, 507-530.

[105] Whelan, C.J. and Dilger, M.L. (1992) Invasive, Exotic Shrubs: A Paradox for Natural Area Managers? Natural Areas Journal, 12, 109-110.

[106] Rodewald, P.G. and Brittingham, M.C. (2004) Stopover Habitats of Landbirds during Fall: Use of Edge-Dominated and Early Successional Forests. Auk, 121, 1040-1055. https://doi.org/10.1642/0004-8038(2004)121[1040:SHOLDF]2.0.CO;2 